\title{
Impact of using a PCM-metal foam composite on charging/discharging process of bundled-tube LHTES units
}

DOI:

10.1016/j.ijheatmasstransfer.2020.119320

\section{Document Version}

Accepted author manuscript

Link to publication record in Manchester Research Explorer

Citation for published version (APA):

Alhusseny, A., Al-Zurfi, N., Nasser, A., Al-Fatlawi, A., \& Aljanabi, M. (2020). Impact of using a PCM-metal foam composite on charging/discharging process of bundled-tube LHTES units. International Journal of Heat and Mass Transfer. https://doi.org/10.1016/j.ijheatmasstransfer.2020.119320

\section{Published in:}

International Journal of Heat and Mass Transfer

\section{Citing this paper}

Please note that where the full-text provided on Manchester Research Explorer is the Author Accepted Manuscript or Proof version this may differ from the final Published version. If citing, it is advised that you check and use the publisher's definitive version.

\section{General rights}

Copyright and moral rights for the publications made accessible in the Research Explorer are retained by the authors and/or other copyright owners and it is a condition of accessing publications that users recognise and abide by the legal requirements associated with these rights.

\section{Takedown policy}

If you believe that this document breaches copyright please refer to the University of Manchester's Takedown Procedures [http://man.ac.uk/04Y6Bo] or contact uml.scholarlycommunications@manchester.ac.uk providing relevant details, so we can investigate your claim.

\section{OPEN ACCESS}




\title{
Manuscript Details
}

\author{
Manuscript number \\ Title
}

Article type
HMT_2019_4971_R1

Effect of rotation on forced convection in wavy wall channels

Full Length Article

\begin{abstract}
In this paper, flow field and heat transfer performance in stationary and rotating wavy channels with different shapes were numerically investigated. Three different geometries were generated through three different values of phase-shift angles of $\varnothing=0,90$ and 180 degrees between the two opposite wavy walls. A cell-centred finite-volume technique was employed to solve the three-dimensional governing equations based on the SIMPLE algorithm technique. Besides, the Menter k- $\omega$ SST turbulence model was used to simulate the turbulent flow in the current study. The wavelength and wave amplitude of the channel examined were $\mathrm{Lw}=20 \mathrm{~mm}$ and $\mathrm{a}=2 \mathrm{~mm}$, respectively. Numerical simulations were carried out over a range of design and operating conditions including the phase-shift angle of $\varnothing=0$ - 180 degrees, Reynolds number of $\operatorname{Re}=1,000-10,000$, and rotating speed of $\Omega=0-1000 \mathrm{rpm}$. The results showed that the surface averaged Nusselt number increases as Re increases for all shapes of the wavy channel, however, at the expense of the raised pressure losses. Also, the wavy channel with a phase-shift of $\emptyset=0$ deg showed the highest enhancement in the performance of heat transfer followed by that of $\emptyset=90$ and $180 \mathrm{deg}$, respectively. The rotation had a strong impact on the flow field and heat transfer performance. With the increase of rotating speed, lower wall heat transfer coefficient significantly increased, while the upper wall heat transfer coefficient exhibited a slight increase, indicating that those three different geometries of the wavy channels had a good versatility at various values of rotating speeds. The numerical results were compared with those available in the literature, and the results were in a good agreement.
\end{abstract}

Keywords

Corresponding Author Nabeel Al-zurfi

Corresponding Author's

Institution

Order of Authors

Suggested reviewers turbulent flow
Wavy channel; Nusselt number; finite-volume method; heat transfer enhancement; Rotation;

Manchester University, Kufa University

Nabeel Al-zurfi, Ahmed Alhusseny, Adel Nasser

Gary Page, H. Al-mithhaji

\section{Submission Files Included in this PDF}

\section{File Name [File Type]}

Cover letter.docx [Cover Letter]

Response to reviewer's comments.doc [Response to Reviewers]

Highlights.docx [Highlights]

Full_paper.docx [Manuscript File]

Conflict of Interest.docx [Conflict of Interest]

AUTHORSHIP STATEMENT.pdf [Author Statement]

To view all the submission files, including those not included in the PDF, click on the manuscript title on your EVISE

Homepage, then click 'Download zip file'.

\section{Research Data Related to this Submission}

There are no linked research data sets for this submission. The following reason is given:

Data will be made available on request 
Dr Nabeel Al-Zurfi

nabeel.al-zurfi@ manchester.ac.uk

Nabeelm.alzurfi@uokufa.edu.iq

International Journal of Heat and Mass Transfer

The current research presents a proposal to enhance the heat transfer performance inside different shapes of a rotating wavy channel. The Menter k- $\omega$ RANS model is employed to simulate the turbulent flow in the present study.

The results of the heat transfer distribution over the wavy surface will be helpful in understanding the physical phenomena regarding the heat transfer coefficient and beneficial in designing more efficiently cooled turbine rotor blades.

It is anticipated that a fair evaluation will be carried out by the reviewers and valuable observations/recommendations will be provided for the improvement of the present or future works.

Sincerely,

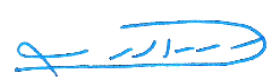

Signature

Dr Nabeel Al-Zurfi 


\section{Summary of the changes have been made:}

- We are thankful and deeply grateful for the efforts of the editor and the reviewers and for their valuable and greats comments which can help us to improve the present work.

\begin{tabular}{|c|c|c|}
\hline & Reviewer's comments & Responses to the comments \\
\hline \multirow[t]{3}{*}{ Reviewer 1} & $\begin{array}{l}\text { 1. The objective of the wavy channels is to } \\
\text { enhance heat transfer in the blade cooling } \\
\text { channels. The authors should also estimate the } \\
\text { normalized Nusselt number Nu/Nu0 for } \\
\text { different Reynolds numbers (to exclude their } \\
\text { effects), as well as the aerothermal efficiency, } \\
\text { which incorporates both the Nusselt number } \\
\text { and the friction factor. In the light of the said } \\
\text { above, I recommend the authors to have a look } \\
\text { at the following papers and the references in } \\
\text { them: Heat and Mass Transfer, 2013, } 49 \text { (5) } \\
679-694 \text { and 2012, } 48 \text { (5) 735-748, as well as } \\
\text { Transactions of the ASME. J. Heat Transfer, } \\
\text { 2011, Vol. 133, No 5, paper 051701. }\end{array}$ & $\begin{array}{l}\text { As requested by the reviewer, additional results have } \\
\text { been presented as listed below: } \\
\text { 1- The normalized Nusselt numbers have been } \\
\text { calculated and discussed. Please see figures } \mathbf{1 7} \text {, } \\
\mathbf{1 8} \text {, and } \mathbf{1 9} \text {. } \\
\text { 2- The aerothermal efficiencies have been } \\
\text { calculated and discussed. Please see figures } \mathbf{2 0} \text {, } \\
\mathbf{2 1 , 2 2} \text {, and } \mathbf{2 3} \text {. }\end{array}$ \\
\hline & $\begin{array}{l}\text { 2. The choice of the turbulence model must be } \\
\text { justified. How can the authors prove that } \\
\text { the k-omega SST model performs better than } \\
\text { the other turbulence models? Several } \\
\text { turbulence models must be compared with } \\
\text { each other, and the final choice must be } \\
\text { justified via a validation of the simulations } \\
\text { with reliable relevant experimental data for the } \\
\text { present geometry. I suggest the authors to take } \\
\text { example from the paper in the Transactions of } \\
\text { the ASME. J. Heat Transfer, 2014, } 136 \text { (5) } \\
\text { Paper 051901, which deals in detail with these } \\
\text { issues. }\end{array}$ & $\begin{array}{l}\text { As requested by the reviewer, several turbulence models } \\
\text { have been compared with each other as shown in Table } \\
\text { 2. } \\
\text { Table } 2 \text { illustrates the comparison of the surface- } \\
\text { averaged Nusselt numbers of the present study with } \\
\text { several RANS turbulence models and the previous } \\
\text { numerical and experimental results of Ahmed et al. [25]. }\end{array}$ \\
\hline & $\begin{array}{l}\text { 3. The language is overall good; an additional } \\
\text { proofreading editing is however } \\
\text { recommended. }\end{array}$ & $\begin{array}{l}\text { The manuscript has been proofread as recommended by } \\
\text { the reviewer to improve the language. }\end{array}$ \\
\hline
\end{tabular}




\begin{tabular}{|c|c|c|}
\hline & $\begin{array}{l}\text { 4. Once the suggested papers are used for the } \\
\text { revision, they must be added to the references } \\
\text { list. }\end{array}$ & $\begin{array}{l}\text { The suggested papers were really useful to make the } \\
\text { requested corrections and enhance the paper quality. } \\
\text { Therefore, the suggested papers have been added to } \\
\text { the references list. Please see Refs. [28, 29, 31, and } \\
\text { 33]. }\end{array}$ \\
\hline \multicolumn{3}{|l|}{ Reviewer 2} \\
\hline & $\begin{array}{l}\text { 1. Why were the rotating-channel simulations } \\
\text { performed for the entire channel length which } \\
\text { includes } 10 \text { wave lengths (fig. 2) and not for a } \\
\text { far smaller section with cyclic boundary } \\
\text { conditions? I can understand the need to } \\
\text { simulate the whole channel for the non- } \\
\text { rotating case since measurements were } \\
\text { available for that case. But for the rotating } \\
\text { flow, where measurements were not available, } \\
\text { simulating the whole channel even when the } \\
\text { flow was periodic seems to be a waste of time } \\
\text { and resources. }\end{array}$ & $\begin{array}{l}\text { We would kindly answer the question as follows: } \\
\text { 1-As the reviewer mentioned in his comment, it was } \\
\text { necessary to simulate the whole channel for the } \\
\text { stationary case because the experimental results were } \\
\text { available for the whole channel. } \\
\text { 2- For the rotating flow case, it was possible to apply } \\
\text { segment periodic boundary conditions instead of } \\
\text { simulating the whole channel in order to significantly } \\
\text { reduce the CPU time and computer memory } \\
\text { requirements. But to make a full comparison and shed } \\
\text { the light upon the difference between the stationary and } \\
\text { rotating flow behavior, the whole channel where } \\
\text { simulated for the case of rotating flow. } \\
\text { 3- The location factor } \mathrm{r} \text { in the centrifugal buoyancy } \\
\text { force term ( } \rho \Omega^{2}(r+x) \beta \Delta T \text { ) is the distance from the } \\
\text { axis of rotation to any point of flow particle in } \\
\text { streamwise direction. The effect of the centrifugal force } \\
\text { when simulating a smaller section would not be } \\
\text { appropriate as the value of } r \text { cannot be set correctly. }\end{array}$ \\
\hline & $\begin{array}{l}\text { 2. Are the rotation effects captured correctly in } \\
\text { the k-omega model? I can see in Eq. } 6 \text { that } \\
\text { rotational terms appear explicitly in the } \\
\text { momentum equations, but in the turbulence } \\
\text { model equations (Eqs. } 4 \text { and 5) no such terms } \\
\text { are present. In the absence of such terms, how } \\
\text { do scalar quantities like } \mathrm{k} \text { and omega respond } \\
\text { to the presence of rotation? Very poorly, I } \\
\text { expect. The authors ought to have established } \\
\text { the appropriateness of using this model in a } \\
\text { rotating flow by comparing with experiments } \\
\text { on isothermal flows in rotating, smooth- } \\
\text { walled channels. }\end{array}$ & $\begin{array}{l}\text { We would kindly answer the questions as follows: } \\
\text { 1- The rotational term appears only in the source } \\
\text { term of the momentum equations. The methods } \\
\text { of adding the Coriolis force term and the } \\
\text { centrifugal force term to the } \mathrm{k} \text {-equation, the } \varepsilon- \\
\text { equation, the } \omega \text {-equation, etc are still } \\
\text { inconspicuously. The couples of papers listed } \\
\text { below show that the effect of Coriolis force and } \\
\text { the centrifugal force were added only to the } \\
\text { source terms of the momentum equations. No } \\
\text { source terms include the rotation forces were } \\
\text { added in the standard and commonly used } \\
\text { turbulence models as shown in those papers. } \\
\text { 2- In the recent years, some studies proposed } \\
\text { turbulence model correction methods and } \\
\text { anisotropic turbulence models for predicting } \\
\text { heat-transfer characteristics in a rotating state. }\end{array}$ \\
\hline
\end{tabular}




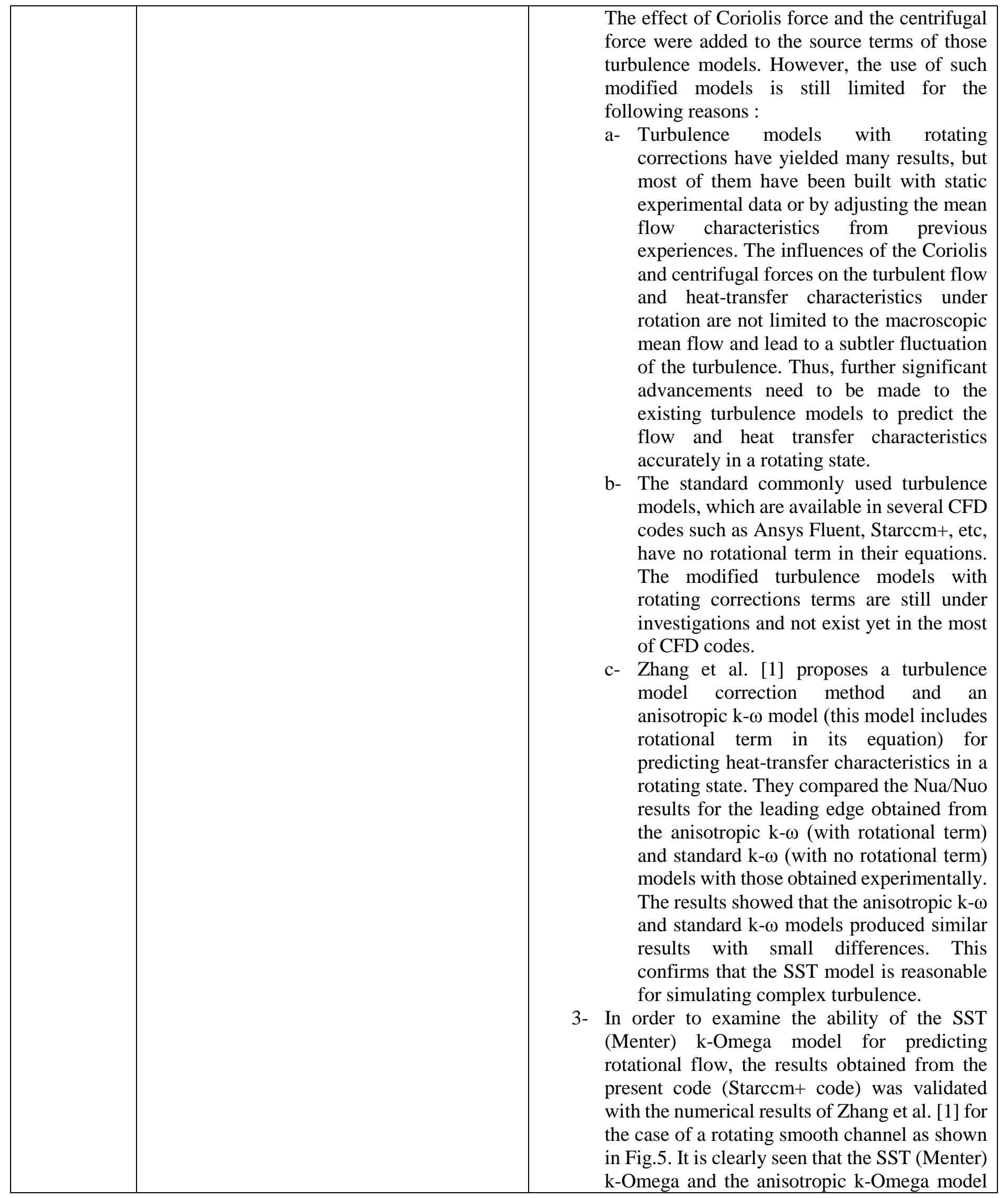




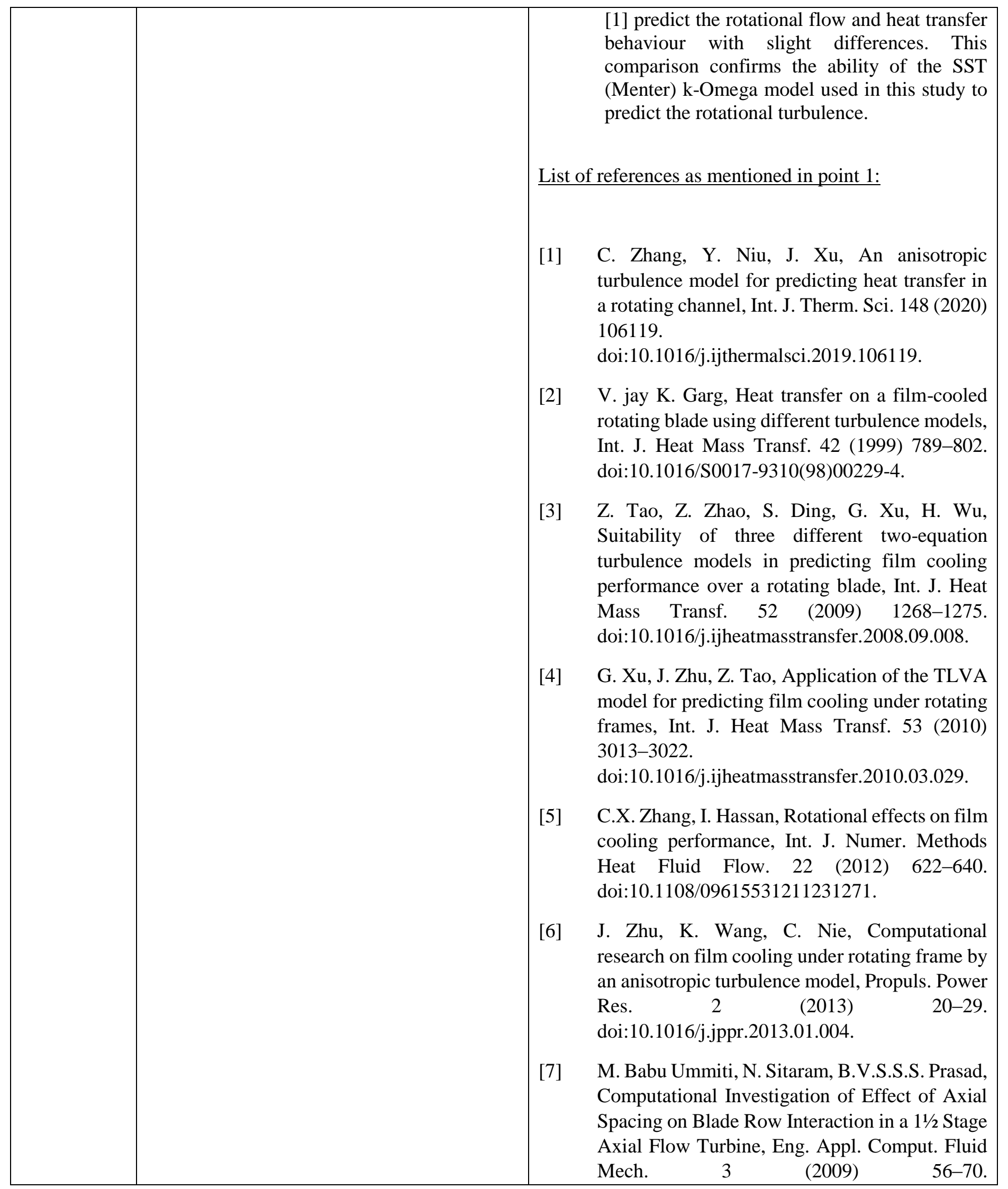




\begin{tabular}{|c|c|}
\hline & doi:10.1080/19942060.2009.11015254. \\
\hline $\begin{array}{l}\text { 3. It is mentioned that the results were } \\
\text { averaged over a "long duration of time". This } \\
\text { is puzzling since the computations were of the } \\
\text { RANS type, not LES. Is this flow supposed to } \\
\text { be unsteady? If so, what is the origin of the } \\
\text { unsteadiness? And what were its effects on the } \\
\text { mean flow and on Nu? Or is it that the flow is } \\
\text { steady but its computations were advanced in } \\
\text { time until a steady-state was achieved? In this } \\
\text { case, time averaging the results would not be } \\
\text { appropriate. }\end{array}$ & $\begin{array}{l}\text { We would kindly answer the question as follows: } \\
\text { As the reviewer mentioned, the flow is steady but its } \\
\text { computations were run for long time until a steady-state } \\
\text { was achieved. The results have been updated and the } \\
\text { "time-averaging", sentence has been removed } \\
\text { throughout the manuscript. }\end{array}$ \\
\hline $\begin{array}{l}\text { 4. The detailed analysis of the results which } \\
\text { takes up the bulk of the discussion is not } \\
\text { justified since no comparisons are made with } \\
\text { data (experimental or simulations), and also } \\
\text { because I doubt that the turbulence model is } \\
\text { good enough to justify such analysis. The } \\
\text { results are, at best, interesting but speculative. }\end{array}$ & $\begin{array}{l}\text { Comparisons have been made with a numerical date for } \\
\text { the case of a rotating smooth channel as shown in figure } \\
5 \text {. }\end{array}$ \\
\hline Minor points: & \\
\hline 1. At 5 pages, the Introduction is far too long. & The introduction section has been reduced \\
\hline $\begin{array}{l}\text { 2. Rotational speed should be non- } \\
\text { dimensionalized. }\end{array}$ & $\begin{array}{l}\text { As the rotation number is } \mathrm{Ro}=\Omega \mathrm{D} / \mathrm{Uin} \text { and } \mathrm{U} \text { value is } \\
\text { varying with Reynolds number, the range of rotation } \\
\text { number (Ro) will be different with each value of Re. It } \\
\text { will be very complicated and confusing to present the } \\
\text { results with multiple ranges of Ro. Thus, we would } \\
\text { kindly recommend keeping the use of dimensional } \\
\text { rotating speed as mentioned above. }\end{array}$ \\
\hline $\begin{array}{l}\text { 3. The captions in Figs. } 6,10 \text { and } 11 \text { are wrong } \\
\text { - the } x \text {-axis is not Reynolds number. }\end{array}$ & The captions in Figs. 6, 10 and 11 have been corrected. \\
\hline
\end{tabular}




\section{Highlights}

- The present work numerically investigates the flow field and heat transfer performance in stationary and rotating wavy channels with different shapes.

- Three different geometries were generated through a desired phase-shift of $\varnothing=0,90$ and 180 degrees between the two opposite sine-wave walls

- A cell-centered finite volume method was employed to solve the time dependent governing equations based on the unsteady SIMPLE algorithm technique

- Results showed that the wavy channel with a shift phase of $\varnothing=0 \mathrm{deg}$ produces the highest surface-averaged Nusselt number and heat transfer enhancement in comparison to other geometries

- Rotation has a strong impact on the flow field and heat transfer performance 


\title{
Effect of rotation on forced convection in wavy wall channels
}

\author{
Nabeel Al-Zurfi ${ }^{a, b, ~}{ }^{,}$, Ahmed Alhusseny ${ }^{a, b}$, and Adel Nasser ${ }^{b}$
}

a. Department of Mechanical Engineering, Faculty of Engineering, University of Kufa, Najaf, Iraq

b. Department of Mechanical, Aerospace and Civil Engineering, School of Engineering, Faculty of Science and Engineering, University of Manchester, Manchester, M13 9PL, UK

*Corresponding Author (email: nabeelm.alzurfi@uokufa.edu.ia)

\begin{abstract}
In this paper, flow field and heat transfer performance in stationary and rotating wavy channels with different shapes were numerically investigated. Three different geometries were generated through three different values of phase-shift angles of $\varnothing=0,90$ and 180 degrees between the two opposite wavy walls. A cell-centred finite-volume technique was employed to solve the three-dimensional governing equations based on the SIMPLE algorithm technique. Besides, the Menter k- $\omega$ SST turbulence model was used to simulate the turbulent flow in the current study. The wavelength and wave amplitude of the channel examined were $\mathrm{L}_{\mathrm{w}}=20 \mathrm{~mm}$ and $\mathrm{a}=2 \mathrm{~mm}$, respectively. Numerical simulations were carried out over a range of design and operating conditions including the phase-shift angle of $\varnothing=0$-180 degrees, Reynolds number of $R e=1,000$ 10,000 , and rotating speed of $\Omega=0-1000 \mathrm{rpm}$. The results showed that the surface-averaged Nusselt number increases as Re increases for all shapes of the wavy channel, however, at the expense of the raised pressure losses. Also, the wavy channel with a phase-shift of $\emptyset=0 \mathrm{deg}$ showed the highest enhancement in the performance of heat transfer followed by that of $\emptyset=90$ and $180 \mathrm{deg}$, respectively. The rotation had a strong impact on the flow field and heat transfer performance. With the increase of rotating speed, lower wall heat transfer coefficient significantly increased, while the upper wall heat transfer coefficient exhibited a slight increase, indicating that those three different geometries of the wavy channels had a good versatility at various values of rotating speeds. The numerical results were compared with those available in the literature, and the results were in a good agreement.
\end{abstract}

KEYWORDS: Wavy channel; Nusselt number; finite-volume method; heat transfer enhancement; Rotation; turbulent flow

\section{Nomenclature}

\begin{tabular}{|c|l|}
\hline$D_{h}$ & hydraulic diameter, $\mathrm{mm}$ \\
\hline$f$ & friction factor \\
\hline$k$ & thermal conductivity, $\mathrm{W} / \mathrm{m} \cdot \mathrm{K}$ \\
\hline$L$ & length of the channel from the inlet to the outlet, $\mathrm{mm}$ \\
\hline $\mathrm{Nu}$ & Nusselt number \\
\hline
\end{tabular}




\begin{tabular}{|c|c|}
\hline $\mathrm{Nu}_{\mathrm{a}}$ & surface-averaged Nusselt number \\
\hline$P$ & pressure, $\mathrm{N} / \mathrm{m}^{2}$ \\
\hline $\mathrm{Pr}$ & Prandtl Number \\
\hline $\operatorname{Pr}_{t}$ & turbulent Prandtl number \\
\hline $\operatorname{Re}$ & Reynolds number \\
\hline RANS & Reynolds averaged Navier-Stokes \\
\hline$t$ & time, $s$ \\
\hline$T$ & fluid temperature, $\mathrm{K}$ \\
\hline$u, v, w$ & velocity components in the $\mathrm{x}, \mathrm{y}, \mathrm{z}$ directions, respectively, $\mathrm{m} / \mathrm{s}$ \\
\hline$y^{+}$ & dimensionless distance of the first point on the wall \\
\hline \multicolumn{2}{|c|}{ Greek letters } \\
\hline$\Delta$ & difference \\
\hline$\eta$ & aerothermal efficiency, $\%$ \\
\hline$\rho$ & fluid density, $\mathrm{kg} / \mathrm{m}^{3}$ \\
\hline $\bar{\mu}$ & dynamic viscosity, N.s $/ \mathrm{m}^{2}$ \\
\hline$\mu_{t}$ & turbulent viscosity, N.s $/ \mathrm{m}^{2}$ \\
\hline$\Omega$ & angular velocity, rpm \\
\hline$\phi$ & phase-shift angle, degree \\
\hline$\beta$ & thermal expansion coefficient, $1 / \mathrm{K}$ \\
\hline \multicolumn{2}{|l|}{ Subscripts } \\
\hline$\infty$ & free stream \\
\hline
\end{tabular}

\section{1-Introduction:}

In the last few decades, researches on the techniques for further enhancement in heat transfer performance for various engineering applications with stringent space restrictions have drawn great attention in research and practice. A wavy surface is one of the classically passive techniques employed in many engineering applications to achieve the industry requirements and demands for greater enhancement in the performance of heat transfer. It is well known that the using of wavy surfaces can substantially enhance the mixing process between the hot fluid close to the wall and the mainstream fluid due to growing separation zones near the wavy walls, which can lead to a better heat transfer performance. Thus, the wavy surfaces work as turbulence promoter intending to enhance heat transfer. One of the great advantages of a wavy surface geometry is that it can be easily structured and produces enhanced performance of fluid flow and heat transfer. The wavy channel structure produces a degree of complexity to the flow compared to a straight channel flow by generating a streamline curvature, recirculation regions, and flow reattachment, producing flow situations that usually exist in relevant geophysical and technical applications. The channels with a wavy wall are types of corrugated surfaces heat transfer exchangers that are utilized for various types of heat transfer applications present in process industries. The investigation of the flow and heat transfer characteristics over wavy surfaces (Fig. 
1(a)) as presented in the work of Dong et al. [1], is a great area of interest for applications in the cooling of electronics devices where a coolant fluid is forced over the boards on which the electronic parts are fixed. Another possible and important application of channel with wavy surfaces is in the internal cooling of a rotating blade in a gas turbine engine as presented in Fig. 1(b). Additionally, wavy surfaces are widely used in other applications such as, manufacturing of polymeric composite, transport processes of biological systems, and in some medical applications that use channels with wavy surfaces.

Many researchers have numerically and experimentally investigated fluid flow and heat transfer process through a corrugated channel. One of the early studies concerning the wavy channel is the work of Goldstein and Sparrow [2] who studied experimentally a turbulent flow and heat transfer in a wavy channel. Their results showed that the wavy channel produces a higher heat transfer coefficient than that of a straight channel for the same values of Re. In the low-Reynolds number turbulent regime, it was found that the rate of heat transfer from the wavy channel is three times larger than for that of a straight channel. Wang and Chen [3] studied numerically the heat transfer for a flow through a wavy channel to investigate the influence of the Reynolds number, Prandtl number, and wavy geometry on Nusselt number and skin-friction coefficient. At higher values of $\mathrm{Re}$, the rate of heat transfer was observed to be significant at a larger value of amplitude wavelength ratio. Nishimura et al. [4] performed an experimental investigation of the heat transfer for a laminar flow inside a symmetric 2D wavy channel. Two different geometries of the channel wall namely, sinusoidal wall and arc-shaped wall were investigated to study the flow and heat transfer behaviours for $\mathrm{Re}=20$ to 300 . The heat transfer rate of the wavy channel was found to be higher than of that from a straight-walled channel.
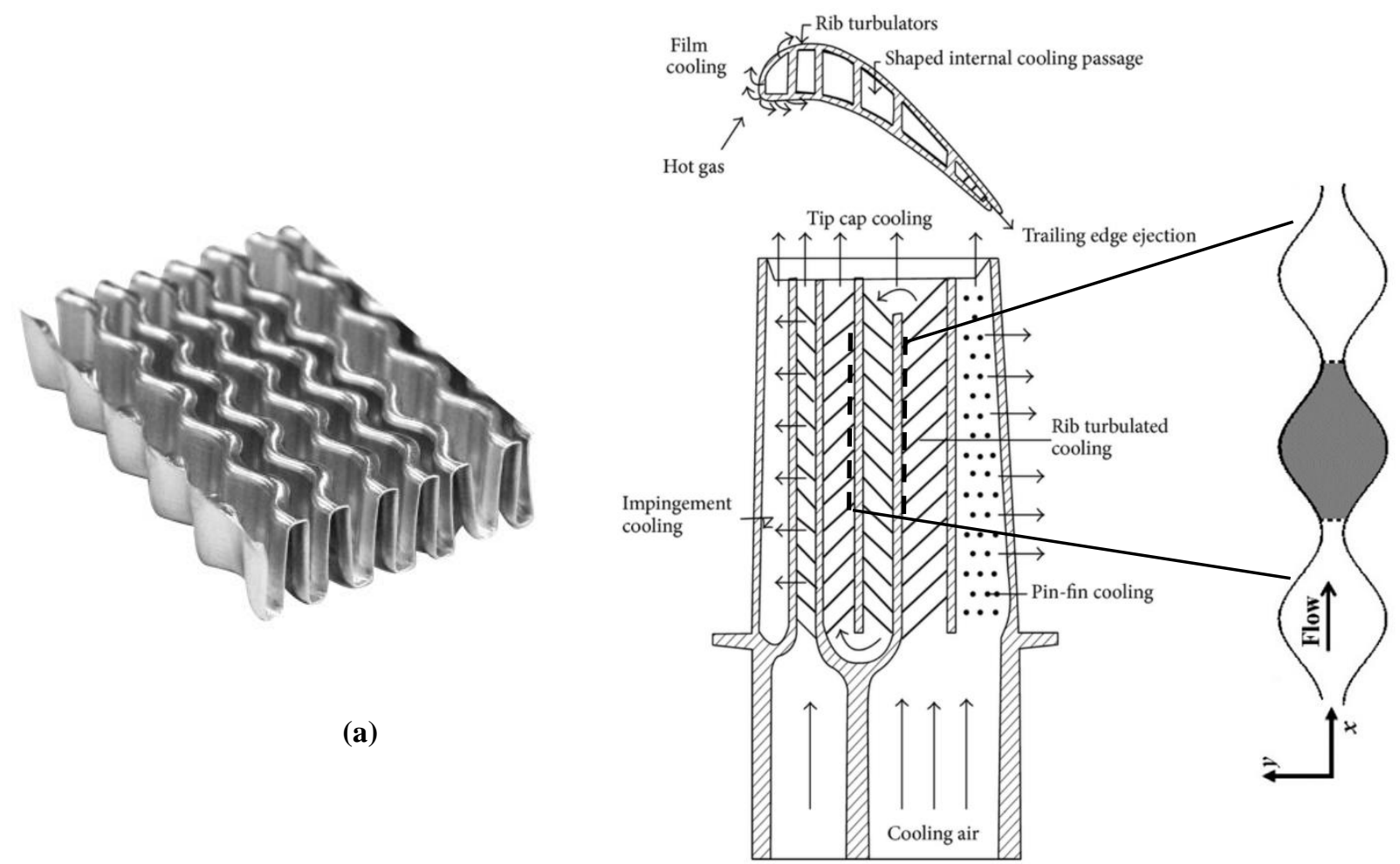

(b)

Figure 1: (a) Photograph of fins with a wavy surface (Dong et al. [1] ); (b) Internal cooling technique for a gas turbine blade. 
Sawyers et al. [5] carried out a numerical study to explore the hydrodynamics effect on the heat transfer coefficient in a corrugated channel. It was found that due to the presence of recirculation zones, heat transfer is higher than that for flat plates. It was also found that a small mean flow in the transverse direction causes enhancement in the heat transfer rate, while a stronger transverse flow leads to a decrease in heat transfer. The experimental study performed by Rush et al. [6] investigated the heat transfer and flow behaviour for a laminar and transition flow in different geometries of wavy walled channels. At low Reynolds numbers, instabilities are manifest near the exit of the wavy channel and move toward the entrance of the channel with an increase in Reynolds number values. Besides, it was observed that increasing flow instability leads to enhancement in the heat transfer rate. Fabbri [7] conducted a numerical study to predict the heat transfer for a laminar flow inside a channel composed of smooth and corrugated walls. The finiteelement method was employed to determine the velocity and temperature distributions. It was found that the heat transfer enhancements of the optimum corrugated profile increase as Re and $\operatorname{Pr}$ numbers increase. Fabbri and Rossi [8] analyzed the heat transfer for a laminar flow in the entrance region of corrugated and flat walls channels. It was observed that the heat transfer was significantly enhanced with an increase in the Reynolds number and corrugated profile amplitude. Naphon [9][11] performed numerical and experimental studies to investigate the heat transfer characteristics and pressure drop of airflow in the corrugated channels. It was found that the corrugated surface has a significant effect on the pressure drop and heat transfer enhancement. Metwally and Manglik [12] performed a numerical study for forced convection heat transfer in wavy channels. It was found that the mixing process generated by transverse vortex cells considerably increases the heat transfer coefficient depending upon the Prandtl number, Reynolds number, and the aspect ratios of the channel. It was also reported that a small friction loss penalty comes with the heat transfer enhancement. Xie et al. [13] studied numerically a fully developed laminar flow in a twodimensional wavy channel. The results showed that both friction factor and overall Nusselt number increase with the increase of Reynolds number. Hossain and Islam [14] carried out a numerical study of a fully developed flow and heat transfer inside a two-dimensional wavy walled channel. It was found that increasing the amplitude or decreasing the channel height leads to a rise in the flow instability and thereby enhance the heat transfer and friction factor. Niceno and Nobile [15] and Haitham et al. [16] compared the performance of a sinusoidal wavy channel with an arc-shaped channel using a numerical simulation. A similar numerical investigation has also been carried out by Ramgadia and Saha [17] where they have made a comparison for the performance of the heat transfer enhancement for three types of channel profiles such as sine-shaped, triangle-shaped, and arc-shaped. Their results showed that the channel with the sinusoidal-wall geometry produces the highest heat transfer coefficient as compared to the arc-shaped and the triangle-shaped geometries. In a recent study of the same authors (Ramgadia and Saha) [18], a numerical study of a fully developed flow and heat transfer performance was carried out through asymmetric wavy-walled geometry. Three different wavy channels geometries were created through three different values of phase shift angles between the upper and lower wavy walls of the channel. The results revealed that the wavy channel geometry with a phase-shift of 0 deg encourages the highest heat transfer compared to the other geometries. Three different types of sinusoidal channel geometries were investigated experimentally by Tolentino et al. [19]. Their results showed that the amount of geometric asymmetry in the configuration leads to an increase in the rate of the mixing process and hence the heat transfer rate. The hydrodynamics and thermal characteristics achieved by simulating laminar flow inside a wavy wall channel were also reported by Haitham [20], Nishimura and Matsune [21], and Mahmud et al. [22]. The dependency of recirculation zone size 
and strength on Reynolds number for a periodic wavy passage was investigated by Haitham et al. [16]. It has also been added that for a periodic staggered wavy channel, Haitham [20], in addition to the effect of the change in Reynolds number, the strength and size of separation region also depends on the distance between the two staggered surfaces. Furthermore, Zhang and Che [23] carried out a numerical investigation to study the effect of a corrugation profile for crosscorrugated plates on the thermal-hydraulic performance. Different types of corrugated plates were examined. The results showed that the flow and heat transfer characteristics of corrugated plates are significantly affected by the corrugation profile. Nusselt number and friction factor for the trapezoidal channel was found to be nearly $400 \%$ higher than for the elliptic channel. Mohammed et al. [24] carried out a numerical study to investigate laminar water flow and heat transfer characteristics in a wavy microchannel. The results showed that the wavy microchannel produces a higher coefficient of heat transfer compared with that of the straight microchannel for the same geometrical parameters. The penalty of pressure loss in the wavy microchannel, however, is much smaller than the enhancement in the performance of heat transfer.

It appears from the aforementioned investigations that the effects of wavy surfaces on the heat transfer performance in corrugated channels have not been studied extensively. No studies are available in the open literature examining the effects of rotation on the flow field and heat transfer characteristics in wavy channels. To the best of the authors' knowledge, the results of the present study are presented for the first time in the open literature for the case of a flow and heat transfer in a rotating wavy channel. The main aims of the present study are to further understand the mechanism for the enhanced heat transfer rate with the use of different geometries of a wavy channel design, optimize a configuration of the corrugated channel, and to explore the effects of rotation on the performance of the heat transfer in corrugated channels for Reynolds numbers ranging from 1,000 to 10,000 using the Reynolds averaged Navier-Stokes (RANS) method. Therefore, the present study aims to numerically investigate the turbulent forced convection flow in rotating wavy channels and to examine the performances of sinusoidal wavy-walled channels with three different values of phase-shift angles of $\varnothing=0,90$ and 180 degrees, Fig. 2 (a-c). The level of enhancement in the Nusselt number due to the use of wavy surfaces is presented and discussed. The results of the flow and heat transfer from wavy walls will be useful to a further understanding of the mechanism of the flow and heat transfer inside corrugated channels and helpful in designing more efficiently cooled rotor blades of gas turbine engines. 


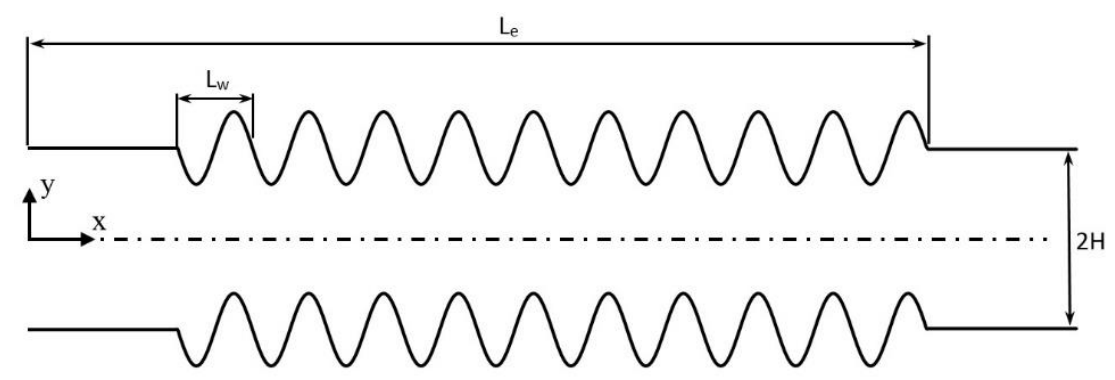

a) $\emptyset=0 \mathrm{deg}$

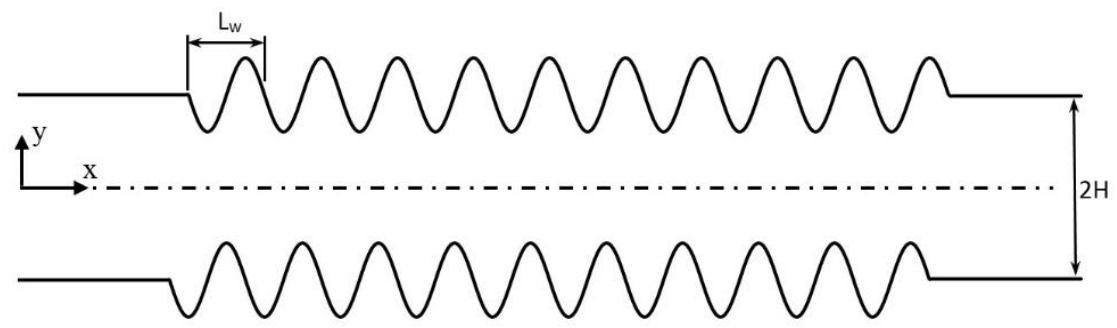

b) $\varnothing=90 \mathrm{deg}$

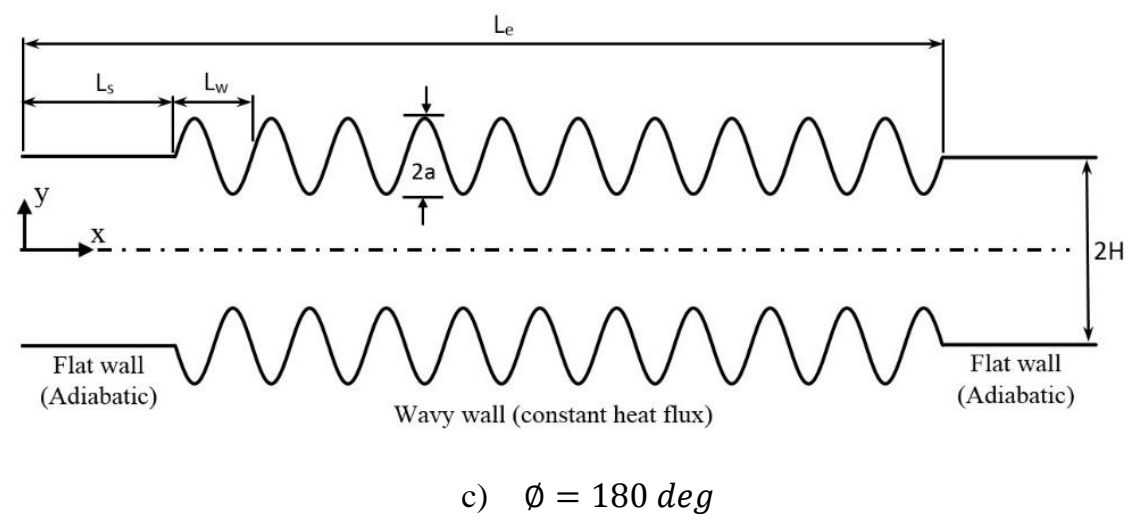

Figure 2: Geometry of the three different types of wavy channels with different phase-shifts angles (a) $\emptyset=0$ deg; (b) $\varnothing=90 \mathrm{deg}$; and (c) $\varnothing=180 \mathrm{deg}$.

\section{2-Mathematical formulation:}

\section{2-1 Problem description:}

The geometry profiles used in the current study are symmetric and asymmetric sinusoidal wavy-walled channel configurations, as shown in Fig. 2. This figure presents a schematic of the three different types of a wavy-walled channel with a detailed description of their geometrical dimensions. The symmetric geometry (Fig. 2(c)) used in this study was derived from the experimental work of Ahmed et al.[25], using exactly the same geometrical parameters as in the experimental setup. The geometry profile, shown in Fig. 2(c), was created using a phase-shift angle 
of $\varnothing=180$ deg between the upper and lower wavy walls of the channel. The profiles of the other two asymmetric wavy channels ( $\varnothing=0$ and 90 degrees) were shaped by displacing the location of the upper wall of the wavy channel with a phase-shift angle of $\varnothing=0$ and 90 degrees, keeping the lower wall fixed as presented in Fig. 2 (a-c). The shape of the wavy walls used in the geometry of the wavy channel within the region of $L_{s} \leq x \leq L_{e}$ was defined by the function $y(x)=H \mp$ $a \sin \left(2 \pi\left(x-L_{s}\right) / L_{w}\right.$, where $a$ is the amplitude of the wavy surface, $x$ is the distance along the $\mathrm{x}$ direction, $L s$ and $L e$ are the start and end points of the wavy-channel wall. $L_{w}$ is the wavelength, and $L$ is the length of the channel from inlet to outlet. Two adiabatic straight ducts with a length of $40 \mathrm{~mm}$ and height of $10 \mathrm{~mm}$ were used as an inlet and outlet sections of the wavy channel to generate appropriate upstream and downstream boundary conditions. As depicted in Fig. 2, the average height $(2 \mathrm{H})$ between the upper and lower walls was $10 \mathrm{~mm}$, the width of the channel $(\mathrm{W})$ was $50 \mathrm{~mm}$, the total length (L) of the entire channel including the two adiabatic sections and wavy duct was $280 \mathrm{~mm}$, the amplitude $(a)$ was $2.0 \mathrm{~mm}$, and the wavelength $\left(\mathrm{L}_{\mathrm{w}}\right)$ was $20 \mathrm{~mm}$. These values are the same as those in the experiment [25].

\section{2-2 Governing equations:}

In the present study, RANS method has been utilized in the modelling of the turbulent flow. The working fluid was assumed to be Newtonian fluid with constant fluid properties except for density and dynamics viscosity, where they were assumed as a function of temperature only (incompressible ideal gas). It was also assumed that the flow and heat transfer are steady, turbulent, fully developed, incompressible and three-dimensional. Consequently, the three-dimensional governing equations for a steady, incompressible, and turbulent flow in terms of tensor notation are [26]:

- Continuity equation:

$$
\frac{\partial}{\partial x_{i}}\left(\rho u_{i}\right)=0
$$

- Momentum equations:

$$
\frac{\partial}{\partial x_{j}}\left(\rho u_{i} u_{j}\right)=-\frac{\partial p}{\partial x_{i}}+\frac{\partial}{\partial x_{j}}\left[\left(\mu+\mu_{t}\right)\left(\frac{\partial u_{i}}{\partial x_{j}}+\frac{\partial u_{j}}{\partial x_{i}}\right)\right]-\frac{2}{3} \frac{\partial}{\partial x_{j}} \rho k \delta_{i j}+S_{\emptyset i}
$$

- Energy equation:

$$
\frac{\partial}{\partial x_{i}}\left(\rho T u_{i}\right)=\frac{\partial}{\partial x_{i}}\left[\left(\frac{\mu}{P r}+\frac{\mu_{t}}{P r_{t}}\right) \frac{\partial T}{\partial x_{i}}\right]
$$


Where $u_{i}$ is the corresponding flow velocity in the i-direction (i corresponds to the system's coordinate directions), $p$ is the fluid pressure, $T$ is the fluid temperature.

To find the turbulent eddy viscosity $\left(\mu_{t}\right)$, the Menter $\mathrm{k}-\omega$ SST turbulence model was employed in the present study as follows [26]:

- Turbulent kinetic energy equation:

$$
\frac{\partial}{\partial x_{i}}\left(\rho k u_{i}\right)=\frac{\partial}{\partial x_{i}}\left[\left(\mu+\frac{\mu_{t}}{\sigma_{k}}\right) \frac{\partial k}{\partial x_{i}}\right]+P_{k}-\beta_{1} \rho k \omega
$$

- The transport equation of $\omega$ for a turbulent flow is as follows:

$$
\begin{gathered}
\frac{\partial}{\partial x_{i}}\left(\rho \omega u_{i}\right)=\frac{\partial}{\partial x_{i}}\left[\left(\mu+\frac{\mu_{t}}{\sigma_{\omega 1}}\right) \frac{\partial \omega}{\partial x_{i}}\right]+\gamma\left(2 \rho \delta_{i j} . \delta_{i j}-\frac{2}{3} \rho \omega \frac{\partial u_{i}}{\partial x_{j}} \delta_{i j}\right) \\
-\beta_{2} \rho \omega^{2}+2 \frac{\rho}{\sigma_{\omega 2} \omega} \frac{\partial k}{\partial x_{k}} \frac{\partial \omega}{\partial x_{k}}
\end{gathered}
$$

Where $S_{\phi i}$ is the source term of the momentum equation, $P_{k}=2 \mu_{t} \delta_{i j} . \delta_{i j}-\frac{2}{3} \rho k \frac{\partial u_{i}}{\partial x_{j}} \delta_{i j} \quad$ is the rate of production of turbulent kinetic energy, and $\delta_{i j}=\frac{1}{2}\left(\frac{\partial u_{i}}{\partial x_{j}}+\frac{\partial u_{j}}{\partial x_{i}}\right)$

Consequently, the turbulent eddy viscosity is defined as [26]:

$\mu_{t}=\rho k / \omega$

The values of the empirical constants and turbulent Prandtl number in equations 1-5 are defined as [26] :

$\sigma_{k}=1.0, \sigma_{\omega 1}=2.0, \sigma_{\omega 2}=1.17, \gamma=0.44, \beta_{1}=0.09, \beta_{2}=0.083, \operatorname{Pr}_{t}=0.9$

To model the rotation effect in a rotating flow field, the multiple reference frame (MRF) model of Luo et al. [27] was adopted by employing the centrifugal and Coriolis forces in the source terms of the momentum equations as follows:

$S_{\emptyset i}=-\rho \varepsilon_{i k j} \varepsilon_{j l m} \Omega_{k} \Omega_{l} X_{m}-2 \rho \varepsilon_{i k j} \Omega_{k} u_{j}$

Where $\varepsilon_{i j k}$ is the Levi-Civita alternating tensor and $\Omega$ is the angular velocity.

The first and second terms on the right-hand side of Eq. 6 are the contributions of the centrifugal and Coriolis forces, respectively. 


\section{2-3 Boundary conditions:}

For the current simulations, the boundary conditions were set as the experimental work of Ahmed et al. [25]. The inlet air velocity value was calculated according to the value of Reynolds number of the cooling air with an angle of flow direction equals to zero. The inlet temperature of the working fluid enters the channels was $25^{\circ} \mathrm{C}$. Turbulence intensity of $5 \%$. At the outlet, the exit static pressure was set equal to zero reference pressure. A convective flux/outflow thermal boundary condition was also assumed at the outlet. The upper and lower wavy walls were subjected to uniform heat flux conditions, while other walls were assumed to be adiabatic. A no-slip smooth wall condition was prescribed on all wall surfaces. The Reynolds number is usually 1,000-10,000 and the rotating speed is generally 0-1000 rpm.

\section{3-Flow and heat transfer characteristics:}

The flow Reynolds number $(\mathrm{Re})$ is defined based on the hydraulic diameter and inlet flow velocity:

$$
R e=\frac{\rho_{\infty} u_{\infty} D_{h}}{\mu_{\infty}}
$$

Where $\rho_{\infty}$ and $\mu_{\infty}$ are the inlet flow density and dynamic viscosity based on the inlet temperature of the flow.

The equation of the convective heat transfer rate was applied to calculate the heat transfer coefficient,

$$
\dot{Q}_{\text {conv. }}=\dot{m} C_{p}\left(T_{f, \text { out }}-T_{f, \text { in }}\right)
$$

Where $\dot{m}, C_{p}, T_{f, \text { in }}$ and $T_{f, \text { out }}$ represent the mass flow rate, specific heat, inlet and outlet bulk temperatures of the working fluid, respectively. Consequently, the local heat transfer coefficient can be defined as follows:

$$
\begin{aligned}
& h=\frac{\dot{Q}_{\text {conv. }}}{A_{c h, f}\left(T_{w}-T_{f}\right)_{L M T D}} \\
& \left(T_{w}-T_{f}\right)_{L M T D}=\frac{\left(T_{w}-T_{f, \text { in }}\right)-\left(T_{w}-T_{f, \text { out }}\right)}{\log \left[\left(T_{w}-T_{f, \text { in }}\right) /\left(T_{w}-T_{f, \text { out }}\right)\right]}
\end{aligned}
$$

Where $T_{w}$ is the local wall temperature, $A_{c h, f}$ is the total heat transfer surface area in contact with the working fluid, and LMTD is the logarithmic mean temperature difference between the wall and the working fluid. Thus, the local Nusselt number $(\mathrm{Nu})$ is calculated from:

$$
N u=\frac{h D_{h}}{k}
$$

The friction coefficient $f$ is employed to evaluate the hydrodynamic performance of the fluid flow inside the wavy channel and is defined as [28], [29] : 


$$
f=\frac{2 \Delta p D_{h}}{\rho U_{\text {in }}^{2} L}
$$

Where $\Delta p$ is the total pressure difference in the wavy channel.

\section{4-Numerical procedure:}

The present numerical simulations were performed using the commercial CFD code STAR-CCM+ (releases 12.02 and 14.02). The discretization of the governing equations in the computational domain was performed using a cell-centred finite volume method in conjunction with the SIMPLE algorithm approach proposed by Patankar and Spalding [30] for the velocitypressure coupling. The second-order upwind difference (SUD) scheme was applied to discretize the convection terms of governing equations, while the second-order central difference (CD) scheme was applied for the diffusion terms. The generated set of the linear algebraic equations was then solved iteratively using the Gauss-Seidel method to find the values of velocity, pressure, temperature and turbulence quantities. The solution technique was coupled with an algebraic multigrid (AMG) solver to advance the convergence. Simulations were run for thousands of iterations to achieve stable statistics of the flow and turbulence. The solution iteration was continued until the convergence criterion reached the residual value of $10^{-5}$ for every component calculated in the entire computational domain. Typical computational time using 64 cores for each case was 48 hours of central process unit time on the multi-processor mode supercomputer in the CSF system of the University of Manchester.

\section{5-Grid independence study:}

To examine the grid independence of the present numerical solution, a preliminary computation for the case of $\mathrm{Re}=10,000$ was performed by using five different grids arrangements with total numbers of 800,000, 1,700,000, 2,700,000, 3,850,000, and 4,926,000 cells. The comparisons were made using the surface-averaged values of lower wall Nusselt number and Y+. Table 1 summarizes grid properties for a typical wavy module. It can be seen from Table 1 that there is no significant change in the value of Nusselt number and y+ when the size of the chosen mesh was increased above 3,850,000 cells. Therefore, the grid with 3,850,000 cells was chosen for the current computations.

Table 1 Comparison of the surface-averaged Nusselt numbers and Y+ values using different grid sizes.

\begin{tabular}{|c|c|c|c|c|c|}
\hline Grids & $\mathbf{8 0 0 , 0 0 0}$ & $\mathbf{1 , 7 0 0 , 0 0 0}$ & $\mathbf{2 , 7 0 0 , 0 0 0}$ & $\mathbf{3 , 8 5 0 , 0 0 0}$ & $\mathbf{4 , 9 2 6 , 0 0 0}$ \\
\hline $\mathbf{N u}_{\mathbf{a}}$ & 255.7 & 268.1 & 283.4 & 285.7 & 285.6 \\
\hline $\mathrm{Y}+$ & 2.12 & 1.54 & 1.01 & 0.82 & 0.79 \\
\hline
\end{tabular}

\section{6-Grid generation:}

A three-dimensions structured, non-orthogonal, and body fitted hexahedral meshes were generated using the Pointwise software (releases V18.0R1) to form the entire computational domain of the channel. This type of meshes is more accurate with less numerical diffusion. The grid was body-fitted to avoid highly skewed grids near the wavy surfaces. The mesh spacing was uniform in the $\mathrm{x}$ - and $\mathrm{z}$ - directions but non-uniform in the y-direction. The spacing of the first grid 
point adjacent to the lower and upper walls was specified with a value of $0.05 \mathrm{~mm}$ and a growth rate (a stretching ratio) of 1.13 to obtain $y+$ less than unity at all locations. This was necessary to satisfy the requirement of the Menter k- $\omega$ RANS model in order to accurately simulate the heat transfer near the walls and viscous sublayer. The computational grids of the present simulation are shown in Fig. 3. The present numerical simulations were carried out using total grid cells of $3,850,000$ nodes, depending on the Nusselt number results obtained from the grid independence study. 

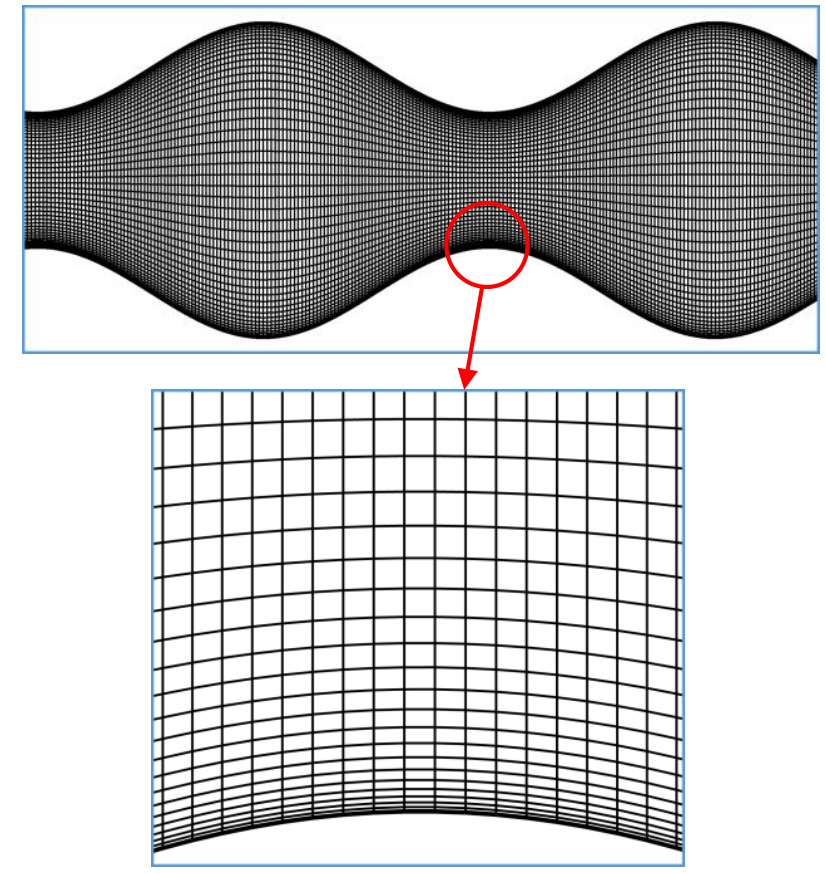

a) $\varnothing=180 \mathrm{deg}$

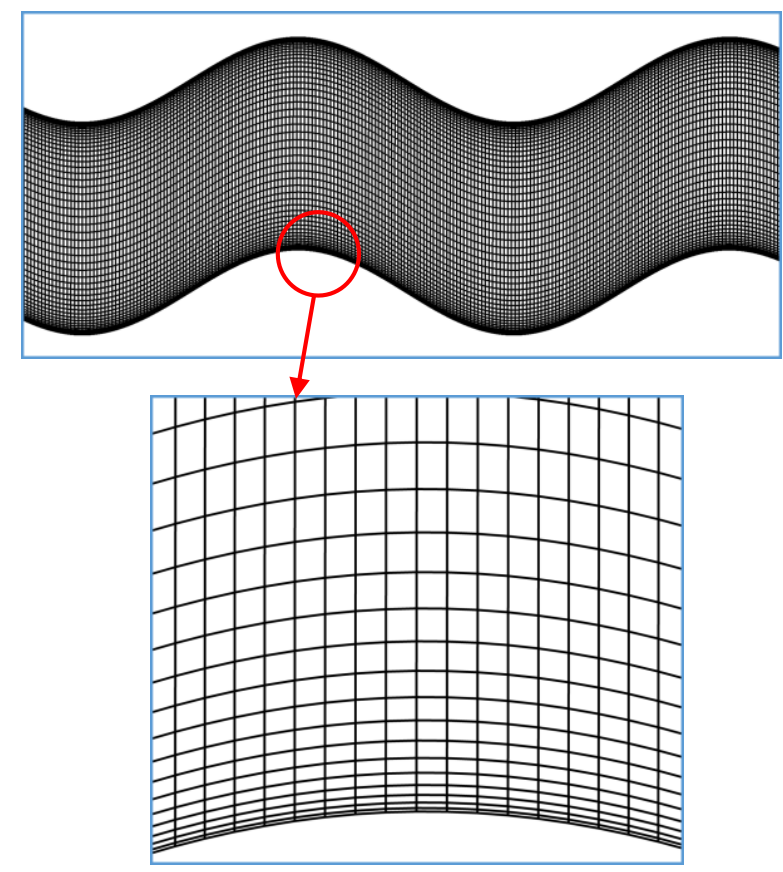

c) $\varnothing=0 \mathrm{deg}$
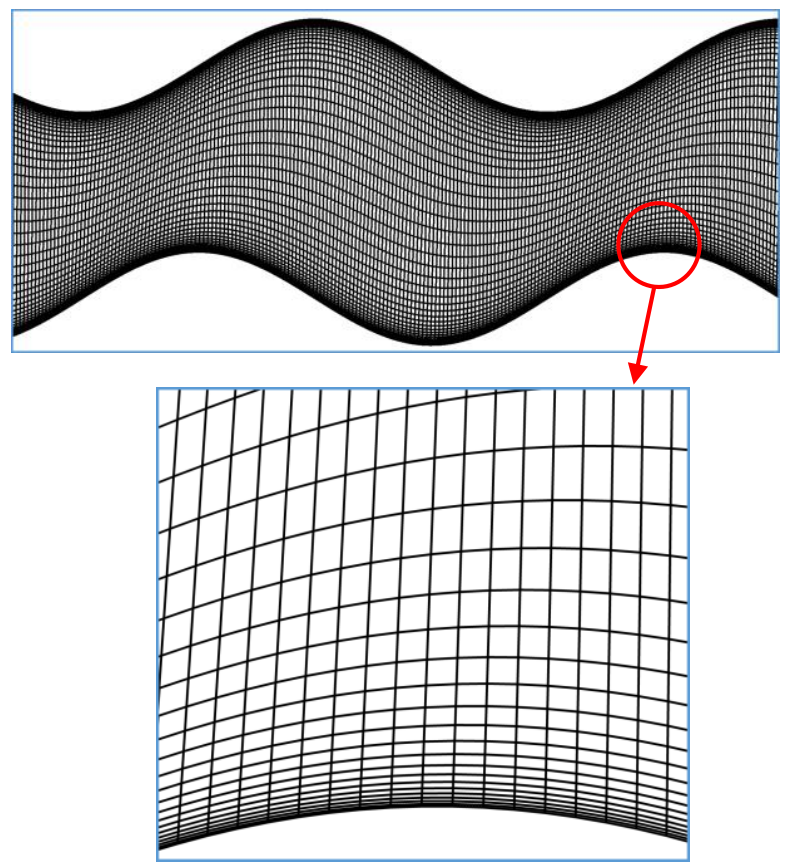

b) $\varnothing=90 \mathrm{deg}$

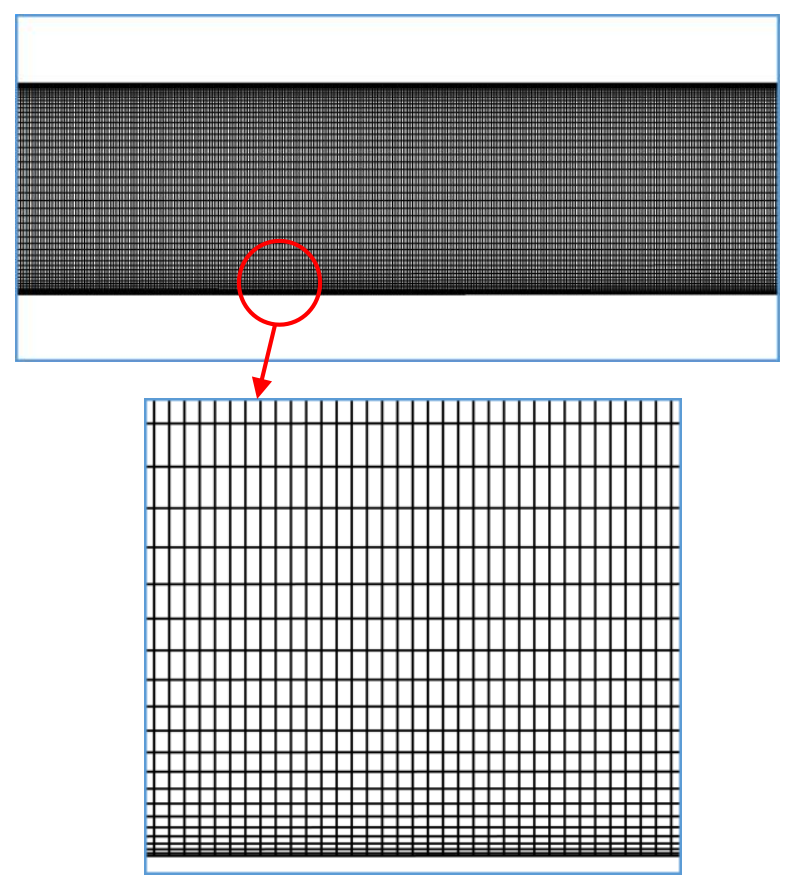

d) straight channel

Figure 3: The computational grids for different types of channel geometry. 


\section{7-Validation of the present numerical method:}

To validate the present numerical results, the surface-averaged Nusselt number and pressure drop for air flowing inside a wavy channel for the case of a stationary condition were simulated using various RANS turbulence models and they then compared with the previous numerical and experimental results of Ahmed et al. [25] as illustrated in Table 2 and Fig. 4. Note that the same boundary conditions were employed in the present simulation to validate the present data with those of Ahmed et al. [25]. Ten commonly used turbulence models were chosen for the present validation as shown in Table 2 in order to select the proper turbulence model for the present numerical simulations [31]. Table 2 shows that the results obtained from the High-Reynolds number Spalart-Allmaras model is the worst as compared with that of the other models. The results from the Standard (Wilcox) k-Omega and SST (Menter) k-Omega models are in a good agreement with the experimental data with maximum relative errors of $4.02 \%$ and $7.66 \%$, respectively. However, SST (Menter) k-Omega model performs slightly better than Standard (Wilcox) k-Omega model. Therefore, the SST (Menter) k-Omega model was employed in the current study to simulate the flow and heat transfer inside wavy chabnnels.

Table 2 Comparison of the surface-averaged Nusselt numbers with several RANS turbulence models and the previous numerical and experimental results of Ahmed et al. [25].

\begin{tabular}{|l|c|c|c|c|c|c|}
\hline & \multicolumn{2}{|c|}{ Re=1000 } & \multicolumn{2}{c|}{ Re=2000 } & \multicolumn{2}{c|}{ Re=4000 } \\
\hline & Nua & Difference \% & Nua & Difference \% & Nua & Difference \% \\
\hline Experimental results of Ahmed et al. [25] & 19.73 & - & 31.84 & - & 85.78 & - \\
\hline Numerical results of Ahmed et al. [25] & 21.11 & - & 33.71 & - & 88.42 & - \\
\hline SST (Menter) k-Omega & 20.48 & $3.80 \%$ & 33.12 & $4.02 \%$ & 87.01 & $1.43 \%$ \\
\hline Standard (Wilcox) k-Omega & 20.61 & $4.46 \%$ & 34.28 & $7.66 \%$ & 87.44 & $1.93 \%$ \\
\hline Realizable k-Epsilon & 21.26 & $7.75 \%$ & 35.84 & $12.56 \%$ & 89.98 & $4.89 \%$ \\
\hline Realizable k-Epsilon two layer & 20.92 & $6.03 \%$ & 34.46 & $8.22 \%$ & 87.67 & $2.20 \%$ \\
\hline Standard k-Epsilon two layer & 21.40 & $8.46 \%$ & 36.35 & $14.16 \%$ & 89.29 & $4.09 \%$ \\
\hline V2F k-Epsilon & 21.73 & $10.13 \%$ & 36.46 & $14.51 \%$ & 89.43 & $4.25 \%$ \\
\hline $\begin{array}{l}\text { Reynolds Stress Turbulence: } \\
\text { Linear pressure strain two layer }\end{array}$ & 21.24 & $7.65 \%$ & 36.13 & $13.47 \%$ & 89.67 & $4.53 \%$ \\
\hline $\begin{array}{l}\text { Reynolds Stress Turbulence: } \\
\text { Quadratic pressure strain }\end{array}$ & 21.69 & $9.93 \%$ & 36.19 & $13.66 \%$ & 90.65 & $5.67 \%$ \\
\hline $\begin{array}{l}\text { Spalart-Allmaras turbulence: } \\
\text { Standard Spalart-Allmaras }\end{array}$ & 21.93 & $11.15 \%$ & 36.96 & $16.08 \%$ & 91.06 & $6.15 \%$ \\
\hline $\begin{array}{l}\text { Spalart-Allmaras turbulence: } \\
\text { High-Reynolds number Spalart-Allmaras }\end{array}$ & 22.05 & $11.75 \%$ & 37.92 & $19.09 \%$ & 94.96 & $10.70 \%$ \\
\hline
\end{tabular}

Furthermore, Fig. 4 shows the comparisons of the surface-averaged Nusselt number profiles and pressure drop of a stationary wavy channel having a phase-shift angle of $\emptyset=180$ with the numerical and experimental results of Ahmed et al. [25] for different values of Reynolds number. These comparisons were carried out to examine the ability of the chosen RANS turbulence model to investigate the characteristics of the flow and heat transfer. From Fig. 4, it is found that the present results are in good agreements with the previous numerical and experimental results. 


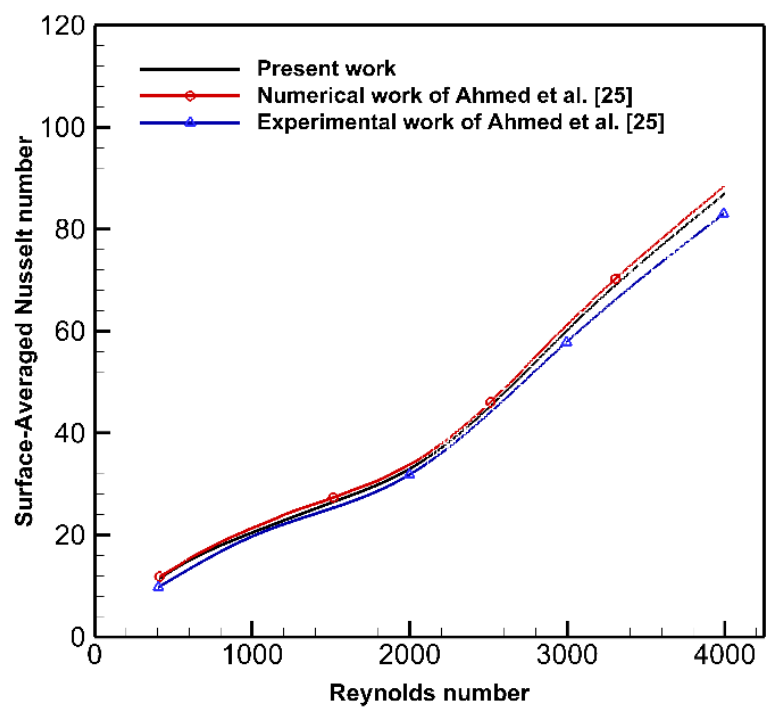

(a)

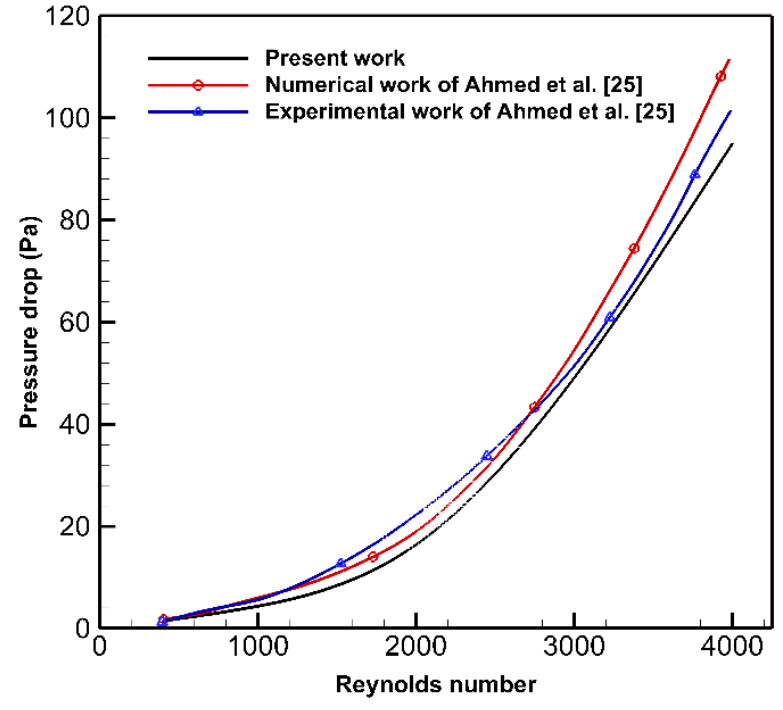

(b)

Figure 4: Comparisons of the present results for (a) surface-averaged Nusselt number, and (b) pressure drop

In order to examine the ability of the SST (Menter) k-Omega model for simulating the rotational flow, the results obtained from the present code (Starccm+ code) were validated with the numerical results of Zhang et al. [32] for the case of a rotating smooth channel under different values of Reynolds number (Re) and Rotation number (Ro). Fig. 5 presents the comparisons of the calculated normalized Nu predicted by the SST (Menter) k-Omega model with those of Zhang et al. [32] predicted by the anisotropic k-Omega model (turbulence model with rotating corrections). It is clearly seen that the SST (Menter) k-Omega model and the anisotropic k-Omega model [32] predict the rotational flow and heat transfer behaviour with slight differences. This comparison confirms the ability of the SST (Menter) k-Omega model used in this study to predict the rotational turbulence. 

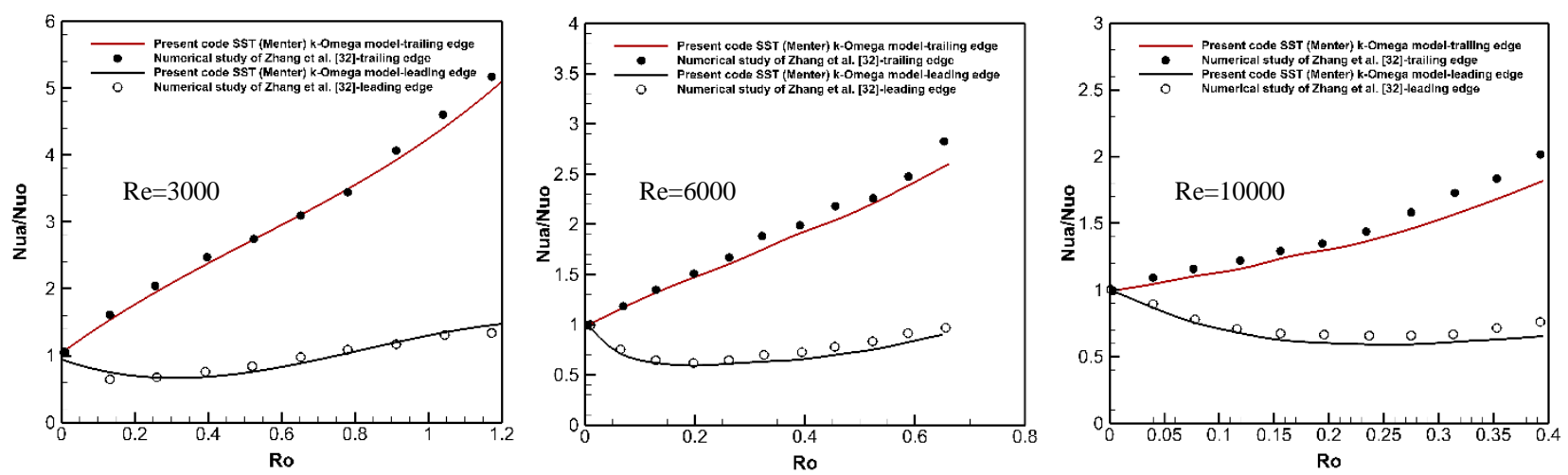

Figure 5: Comparisons of the calculated normalized Nusselt numbers of a rotating smooth channel with those of Zhang et al. [32]

\section{8-Results and discussions:}

Fig. 6 presents the variation of the surface-averaged Nusselt number with Reynolds number for the three different geometries of the wavy channels and the straight channel at both lower and upper walls with different values of rotating speed. In general, the surface-averaged Nusselt number increases with an increase in the value of Reynolds number for all shapes of the channels because of increasing the Reynolds number can increase the temperature gradient on the walls of the channel. It is also obvious from the figure that the wavy channel with a phase angle of $\varnothing=$ 0 deg provides the highest value of Nusselt number among the four configurations at all rotating speeds, while the straight channel provides the lowest value of Nusselt number at the same Re and $\Omega$. This behaviour is expected because the separation zones generated in all geometries of wavy channels can significantly enhance the fluid-mixing process within these channels. It reveals that re-circulation zones generated by the wavy unit play an important role in the flow behaviour and heat transfer. Furthermore, the wavy unit acts as a bulkhead, restricting the fluid spanwise spreading at the near wall. It is obvious that the substantial impact of the wavy wall on working fluid is diversion rather than separation. The re-circulation zone generated by the tip of the wavy unit is mainly active in the gap behind the way unit tip. This gap further limits its lateral movement. Due to the diversion caused by the wavy unit, the fluid has a substantial impact on wall heat transfer, particularly in the first wavy units of the channel wall. This is the reason why the heat transfer rate is at the highest level at the entrance of the channel as shown in Fig. 7. When the fluid reaches the tip of the wavy unit, the cross-sectional area of the flow becomes smaller and thus the fluid velocity increases. As a result of this, no flow separation can be seen in the area of the wavy unit. In the wavy unit vicinity, the secondary flow induced in the tip of the wavy unit becomes the main flow pattern. Consequently, the wavy unit may enhance the heat transfer rate from the wavy channel as shown in Fig. 6. A similar trend is observed for Nusselt numbers at all rotating speeds. 

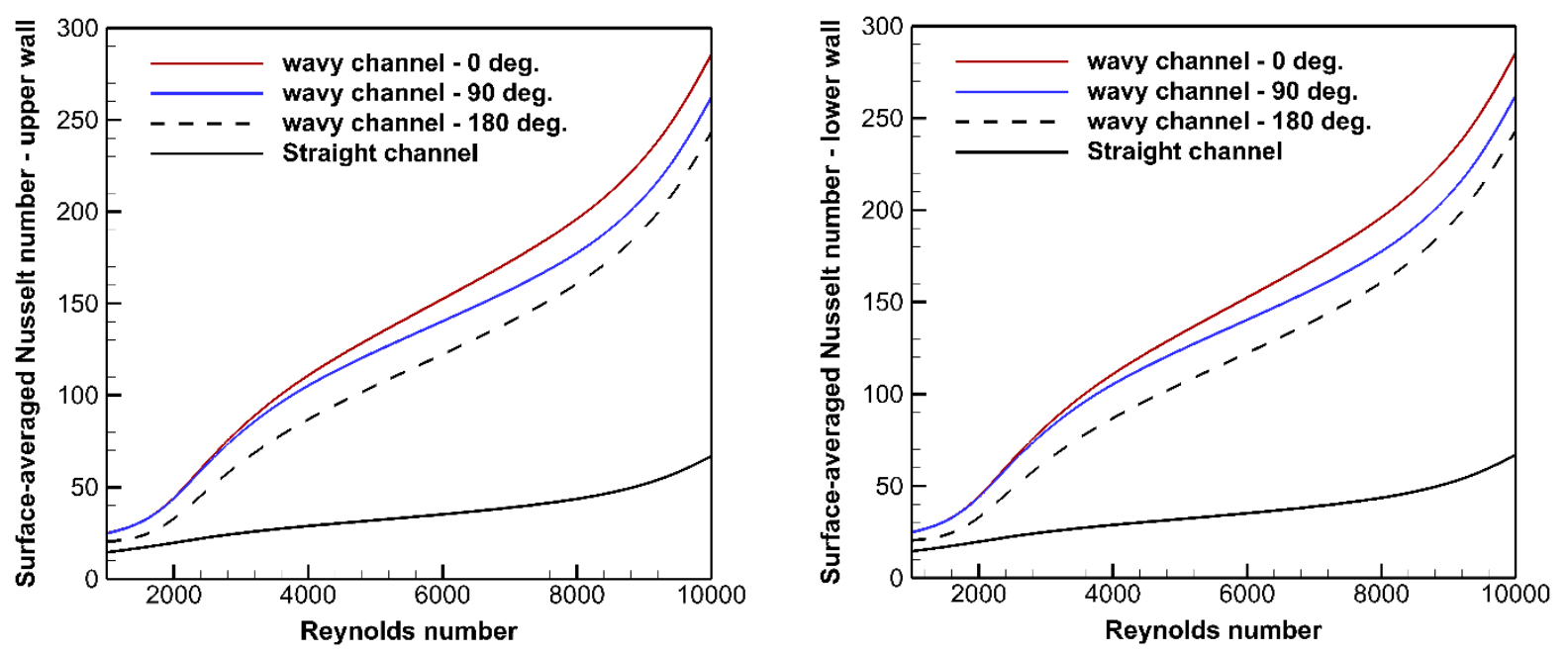

Figure 6: Surface-averaged Nusselt number as a function of Reynolds number for different shapes of the channels at $\Omega=0$.

The variation of the local Nusselt numbers at $\mathrm{Z}=0, \Omega=0$, and $\mathrm{Re}=2000$ along the streamwise direction for the four different types of studied channels is presented in Fig. 7. The figure illustrates that the wavy channel with a phase shift angle of $\phi=0$ has the highest enhancement in the performance of heat transfer among the fourth configurations. In addition, the value of Nusselt number for $\phi=0$ has also the highest fluctuation amplitude. Comparison of the variation of local Nusselt number clearly shows that the wavy channel with a phase shift angle of $\phi=0$ has a higher frequency of oscillations. It can be concluded that the flow with higher frequencies provides a higher heat transfer rate due to the enhanced mixing process between the hot fluid near the walls and the mainstream fluid. Fig. 7 reveals that the Nusselt number profiles of the wavy channels with $\phi=0$ and 90 degs follow the Nusselt number profile of the wavy channel with $\phi=180$. At all locations, it appears that the highest values of the Nusselt number are for the wavy channel with $\phi=0$ whereas the lowest values are produced by the wavy channel with $\phi=180$. 

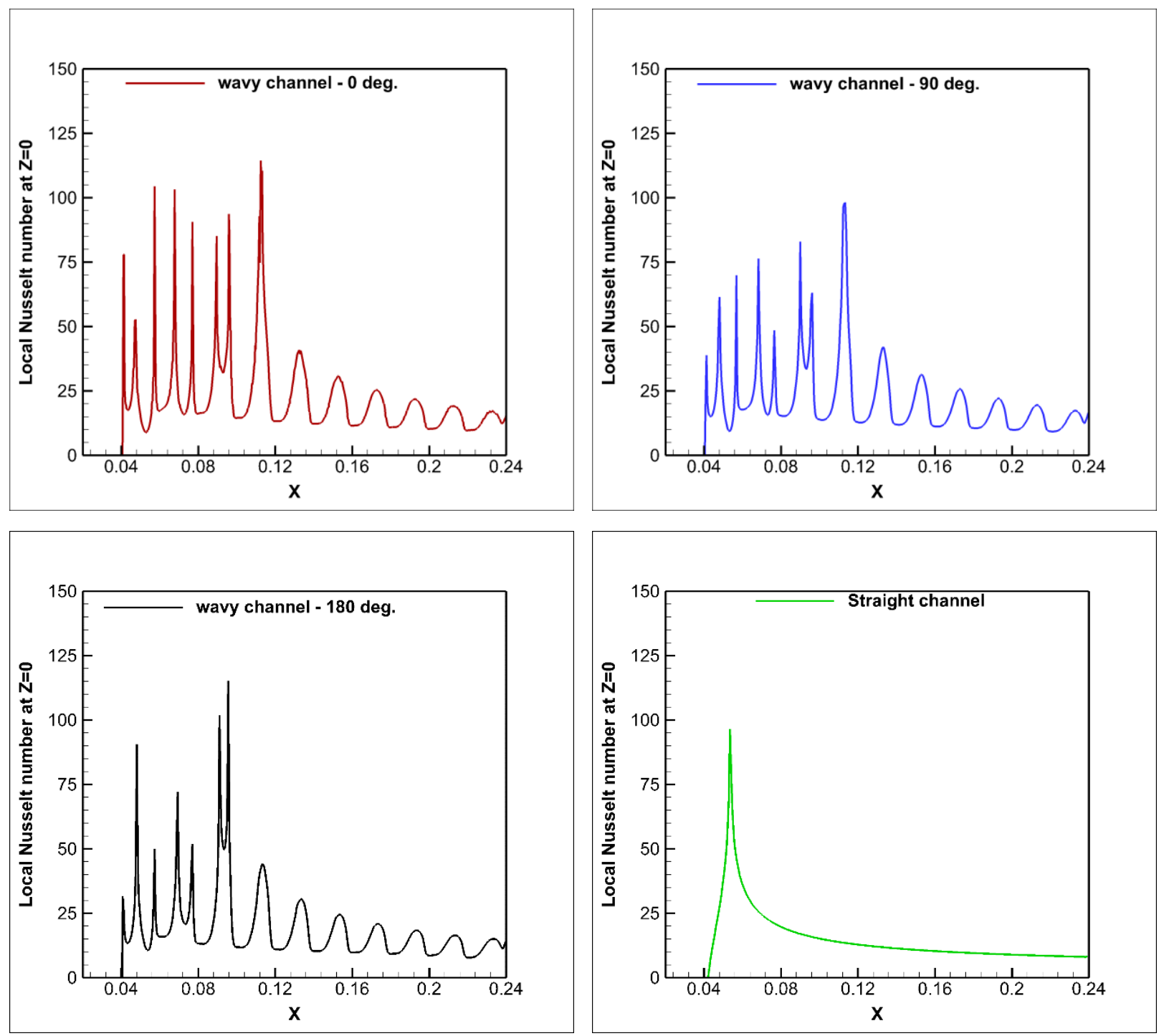

Figure 7: Local Nusselt number distributions on the upper/lower wall along the streamwise direction for different shapes of channel at $\mathbf{\Omega}=\mathbf{0}$.

Both lower and upper walls of the wavy channels exhibit a similar trend in the variation of local Nusselt number but with a phase shift. It is obvious from the figures that the position at which $\mathrm{Nu}$ changes its sign corresponds to the position at which the flow separates or reattaches at the wavy wall of the channel. The low value of the local Nusselt number shows that the heat transfer process happening exactly after the tip of the wavy unit is very low. This is to be expected because the mixing process between the trapped fluids in the secondary flow regions with the core of the cooling fluid is minimal. At $\mathrm{X}<0.08 \mathrm{~m}$, the heat transfer rate is the highest as the thickness of the boundary layer in this location is thinner. Under the stationary condition, the wavy unit causes a diversion impact on the working fluid and discourages its vertical movement. Thus, the mixing process between the hot fluid close to the wall and mainstream cold fluid is extremely weak, particularly within the first wavy units. This can help to promote the characteristics of the flow, however, it is not conducive to the enhancement in heat transfer. 

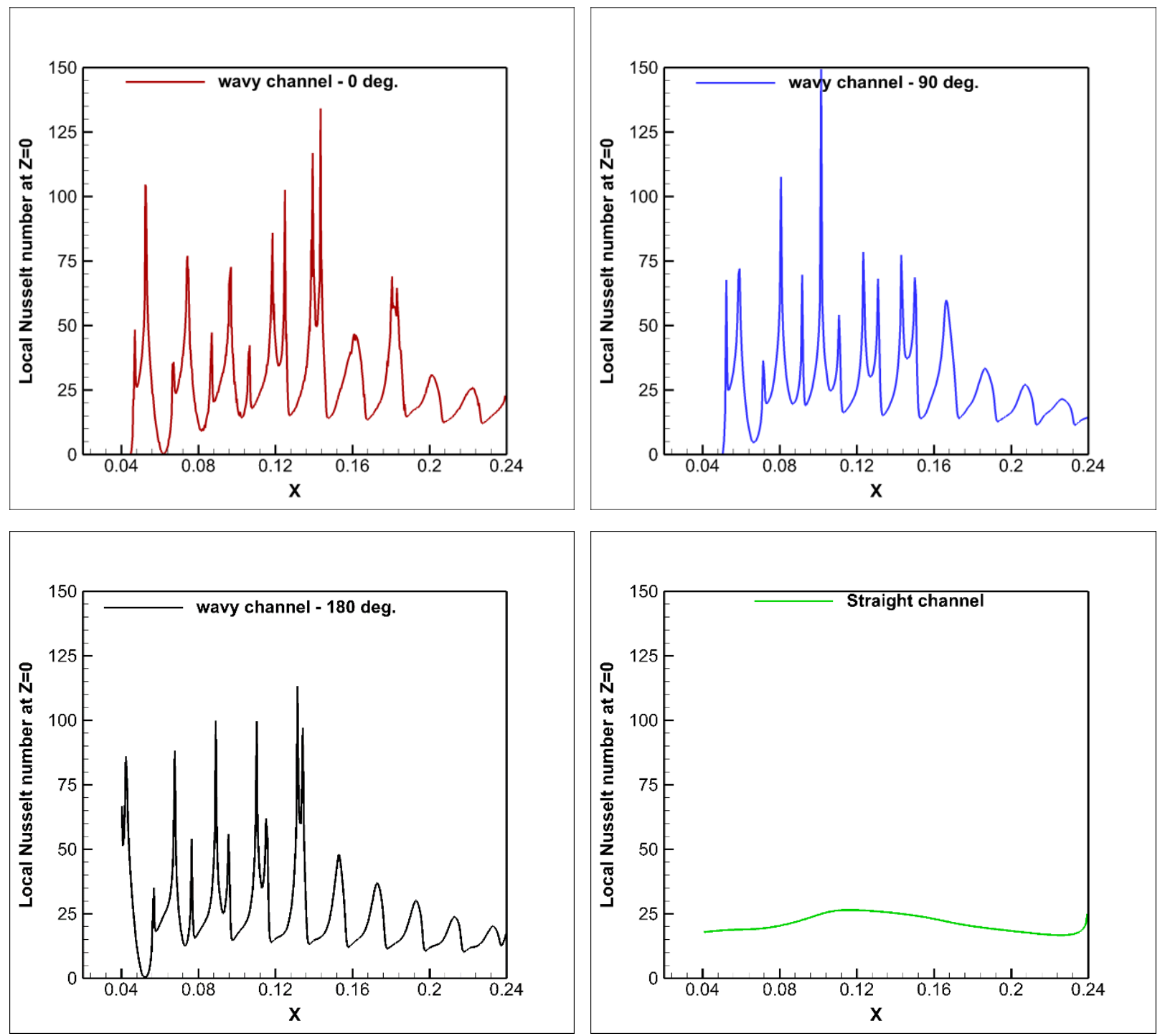

Figure 8: Local Nusselt number distributions on the upper wall along the streamwise direction for different shapes of channels at $\Omega=\mathbf{1 0 0 0}$.

In the stationary case, the values of Nusselt number on both lower and upper walls are similar. But in the rotation case, Nusselt numbers on both walls are different. Figs. 8 and 9 present the variations of the local Nusselt numbers at $\mathrm{Z}=0, \Omega=1000$, and $\mathrm{Re}=2000$ along the streamwise direction for different geometries of the studied channels. The difference in Nusselt number values on the lower and upper walls is due to the contributions of the centrifugal buoyancy force and Coriolis force. These two forces have a substantial effect on the flow and heat transfer characteristics. When the channel rotates in the anti-clockwise direction, the induced centrifugal buoyancy force $\left(\rho \Omega^{2}(r+x) \beta \Delta T\right)$ and Coriolis force $(2 \rho \Omega \mathrm{v})$ would act in the flow direction while the Coriolis force $(-2 \rho \Omega \mathrm{u})$ would act in the negative y direction as shown in Fig. 10. On the other hand, the centrifugal buoyancy force is weaker than the Coriolis force as the variation in the 
temperature of the working fluid inside the channel is very low. Consequently, the centrifugal buoyancy force has no obvious effect on the flow and heat transfer process.
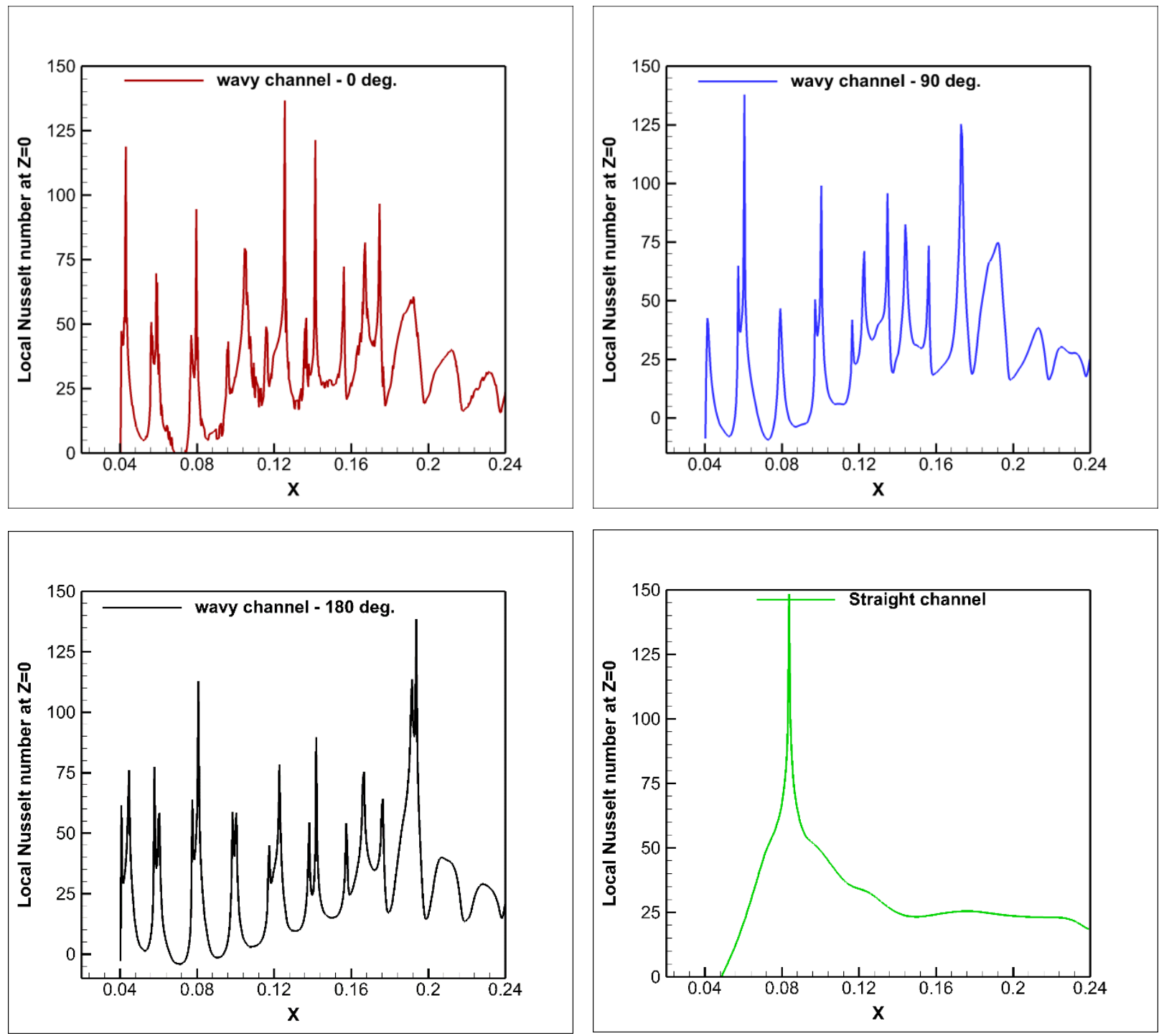

Figure 9: Local Nusselt number distributions on the lower wall along the streamwise direction for different shapes of channels at $\Omega=1000$. 


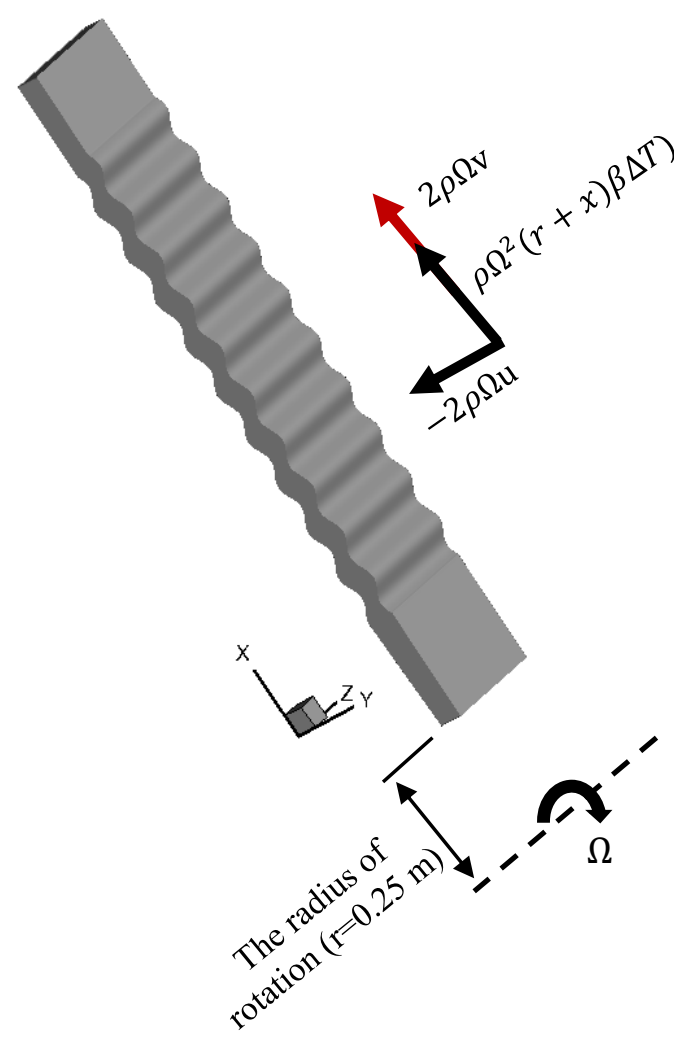

Figure 10: Force analysis of the flow inside a rotating channel.

Under the rotating condition, the Coriolis force $(-2 \rho \Omega u)$ pushes the mainstream fluid toward the lower wall of the channel and then intensifies the fluid mixing and enhances the mass exchange. Moreover, this is in favour of significantly increasing the Nusselt number of the lower wall but it decreasing (at low Re) or slightly increasing (at high Re) the Nusselt number on the upper wall depends on the value of Re as the flow detached from the upper wall due to the effect of the Coriolis force $(-2 \rho \Omega \mathrm{u})$. Downstream of the wavy unit (after the groove), the Coriolis force $(2 \rho \Omega \mathrm{v})$ further increases the fluid turbulent kinetic energy. In addition, the Coriolis force has a significant impact on the lower wall, pushing the impingement's stagnation point within the first wavy units to move toward the wavy wall. The effects of rotation are laid out in Figs. 11 and 12. Some differences can be clearly seen in the lower and upper walls Nusselt number values. 

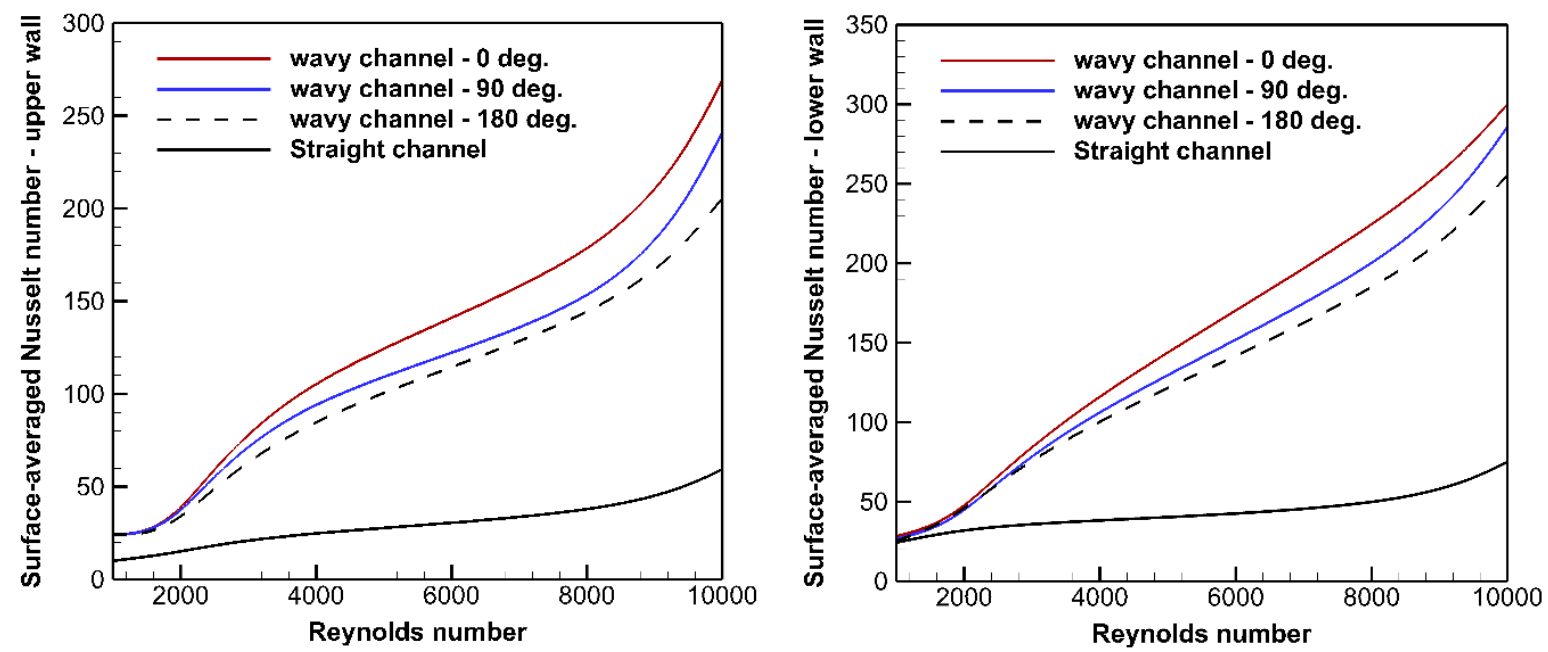

Figure 11: Surface-averaged Nusselt number as a function of Reynolds number for various shapes of channels at $\Omega=500$ rpm.
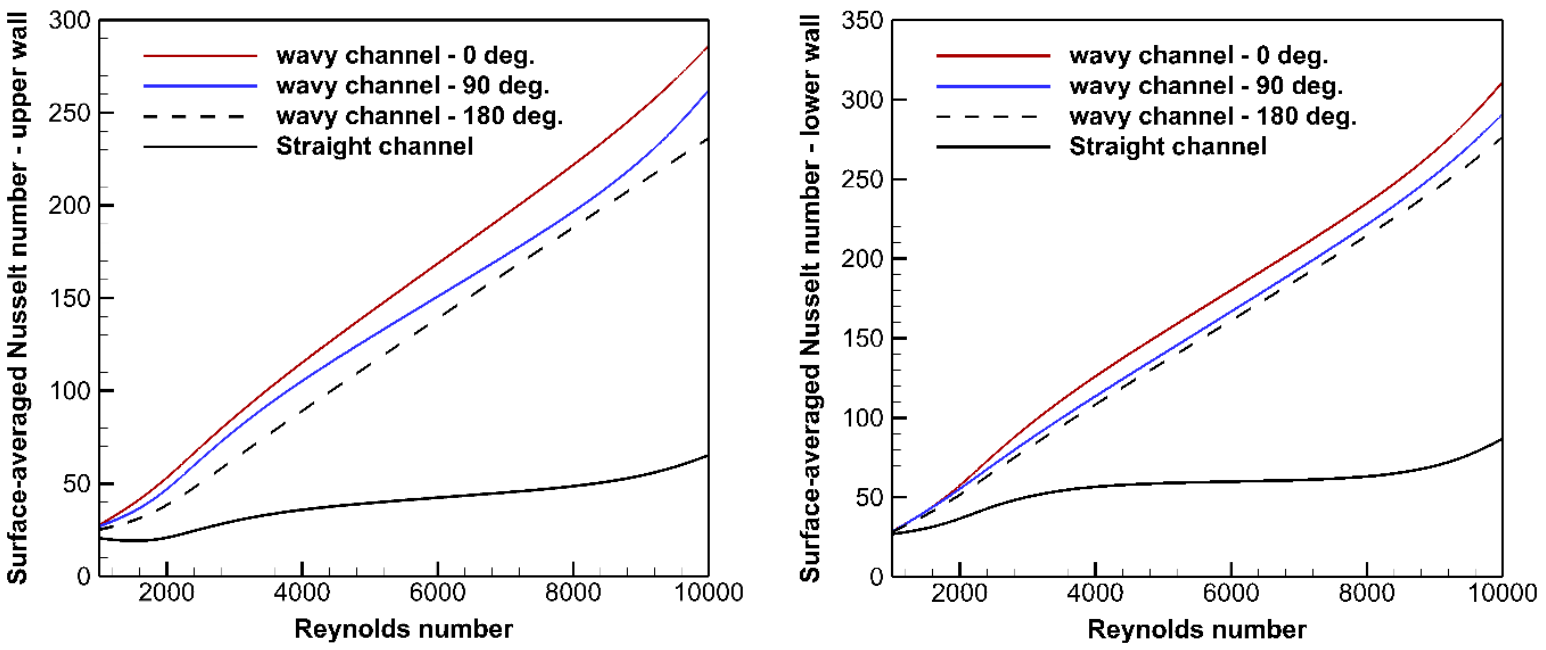

Figure 12: Surface-averaged Nusselt number as a function of Reynolds number for various shapes of channels at $\Omega=1000 \mathrm{rpm}$. 
The velocity field in the display mode of line integral convolution for the wavy channel geometries with phase-shifts angle values of $\emptyset=0,90$, and 180 degrees are illustrated in Fig. 13. The flow evolution zones in both grooves (wavy unit section) of the wavy channel for both $\varnothing=0$ and $\varnothing=90$ degrees are the same as that of the wavy channel with $\emptyset=180$ degree, showing the generation of secondary flow regions in both grooves of the channel. It is clearly seen from the figure that there is no obvious mixing between the secondary flow zones formed in the grooves on the upper and lower walls at any location inside the channel. However, the figure also shows that the flow field distribution is asymmetrical about the centerline of the channel due to geometric asymmetry. This is because of the fact that the space between the upper and lower groves is quite large and the fluid stream between the upper and lower secondary zones is stable. However, momentum transfer between fluids in the upper and lower grooves is possible due to the differential pressure. Channels with smaller space between the two walls may lead to a significant mixing between the secondary flows zones created on the two walls. The asymmetry in the flow rises with the geometric asymmetry and the highest asymmetry in the flow appears in channels with a shift phase angle of $\varnothing=0$. The flow structures through the channels with phase shift angles of $\emptyset=0$ and 90 degrees are quite similar to that of the channel with $\emptyset=180$ degree, showing generation of a single secondary flow zone in the both grooves of the channel. For the channel with $\varnothing=0$, the existence of a pair of secondary flow zones in each groove causes a substantial mixing process of the fluids and thence increasing the rate of heat transfer. The flow structures behaviours through the wavy channels indicate that the phase-shift angle has a substantial impact on the flow characteristics and consequently the heat transfer coefficient. When the channel at stationary conditions, there is a separation accumulation phenomenon on the upper and lower wavy walls of the channel. When the channel rotates, the Coriolis force and centrifugal force have multiple impacts on the flow field and heat transfer. These forces reduce the adverse pressure gradient upstream of the groove tips and significantly reduces the recirculation bubble in the flow region of a minimum cross-section. These forces also weaken the low-energy fluid in regions close to the side walls of the channel, especially downstream of the channel, which promotes the performance of heat transfer. In addition, these forces significantly increase the turbulent kinetic energy of the working fluid, which may explain why the heat transfer rate rapidly increases at a high Re. Consequently, rotation is beneficial to enhance the mass exchange between the mainstream cooling air and hot fluid and thus leads to a significant heat transfer enhancement, especially on the lower wall compared with that of the stationary case. 

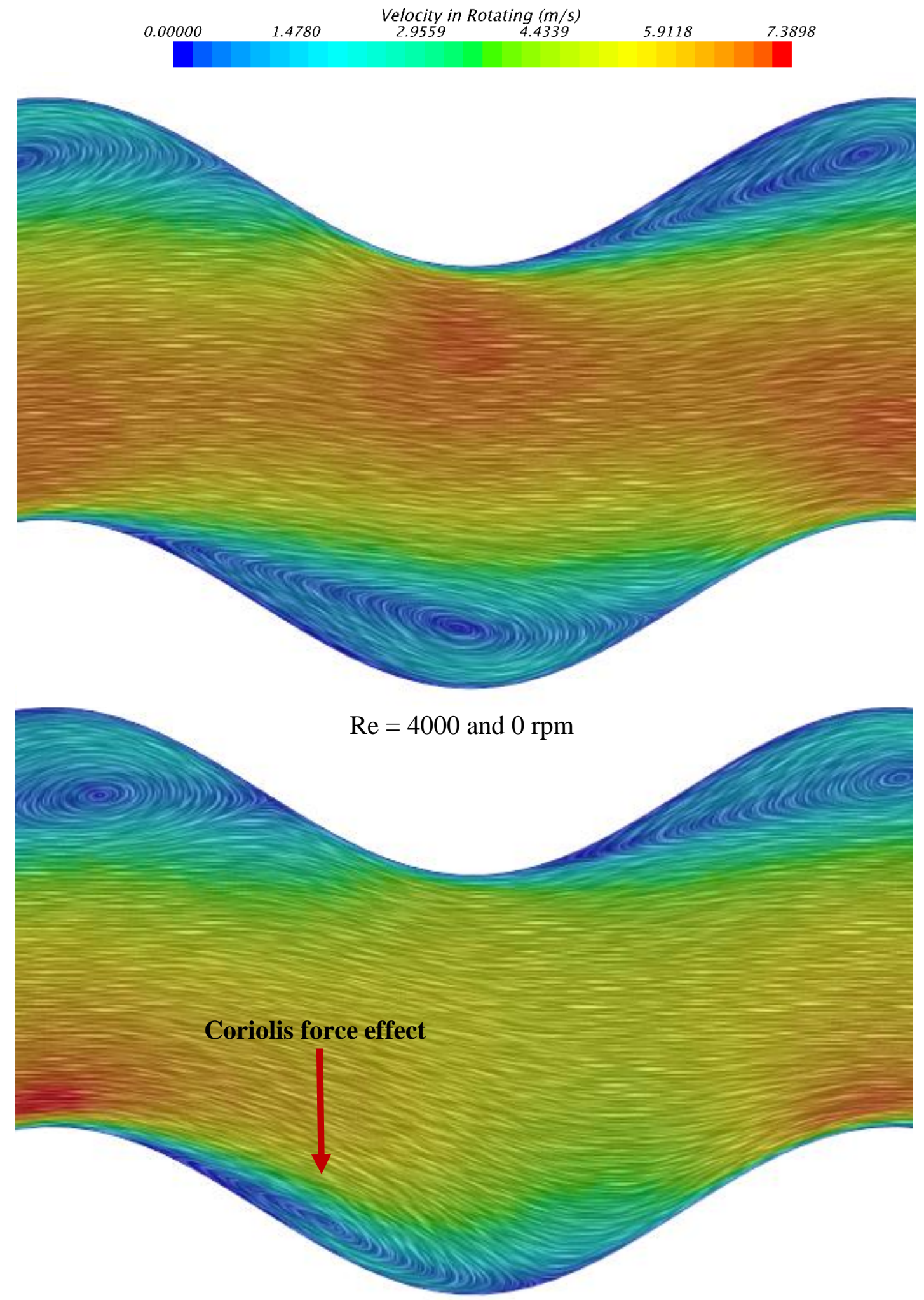

$\mathrm{Re}=4000$ and $1000 \mathrm{rpm}$

a) $\varnothing=0 \mathrm{deg}$ 


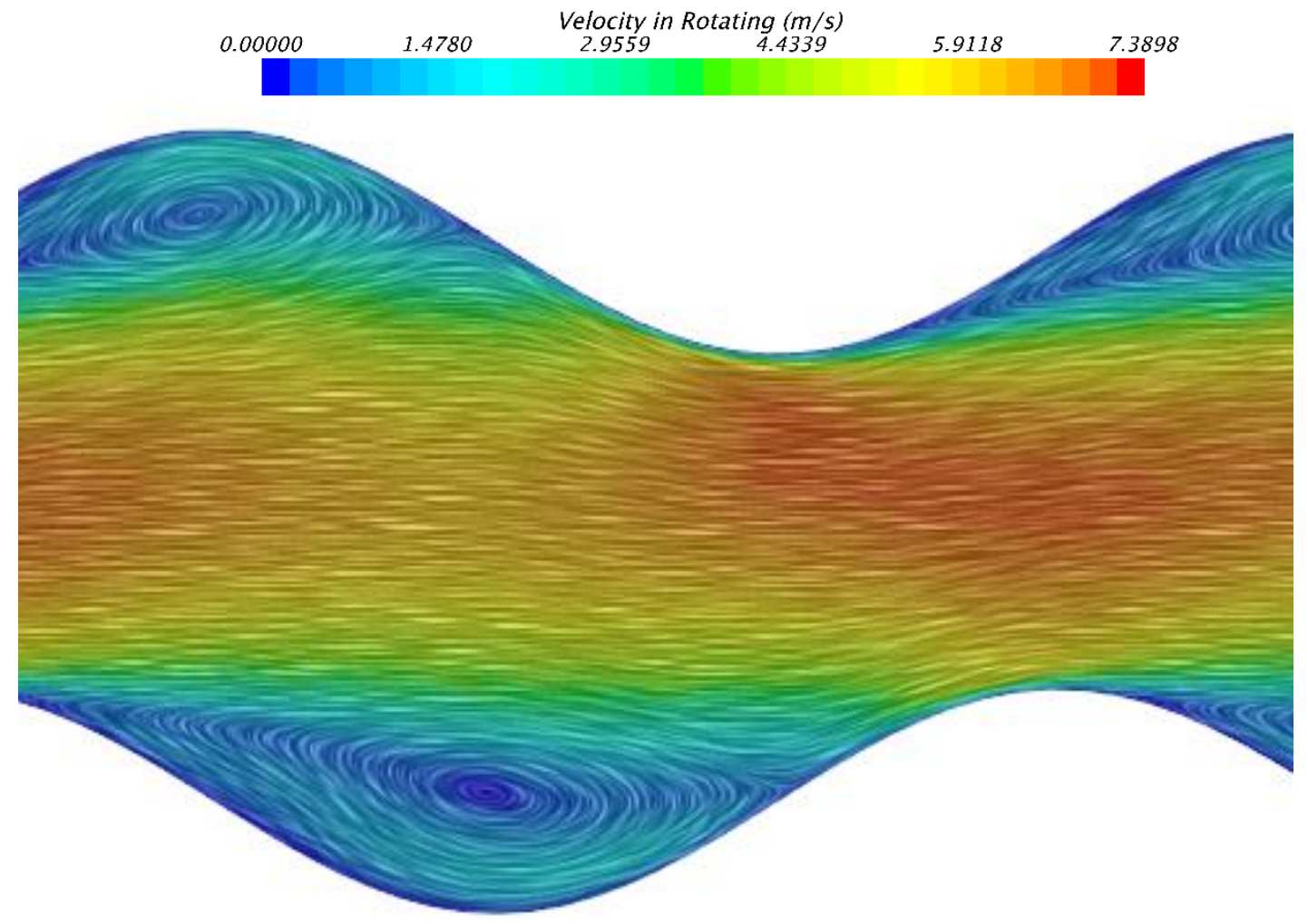

$$
\mathrm{Re}=4000 \text { and } 0 \mathrm{rpm}
$$

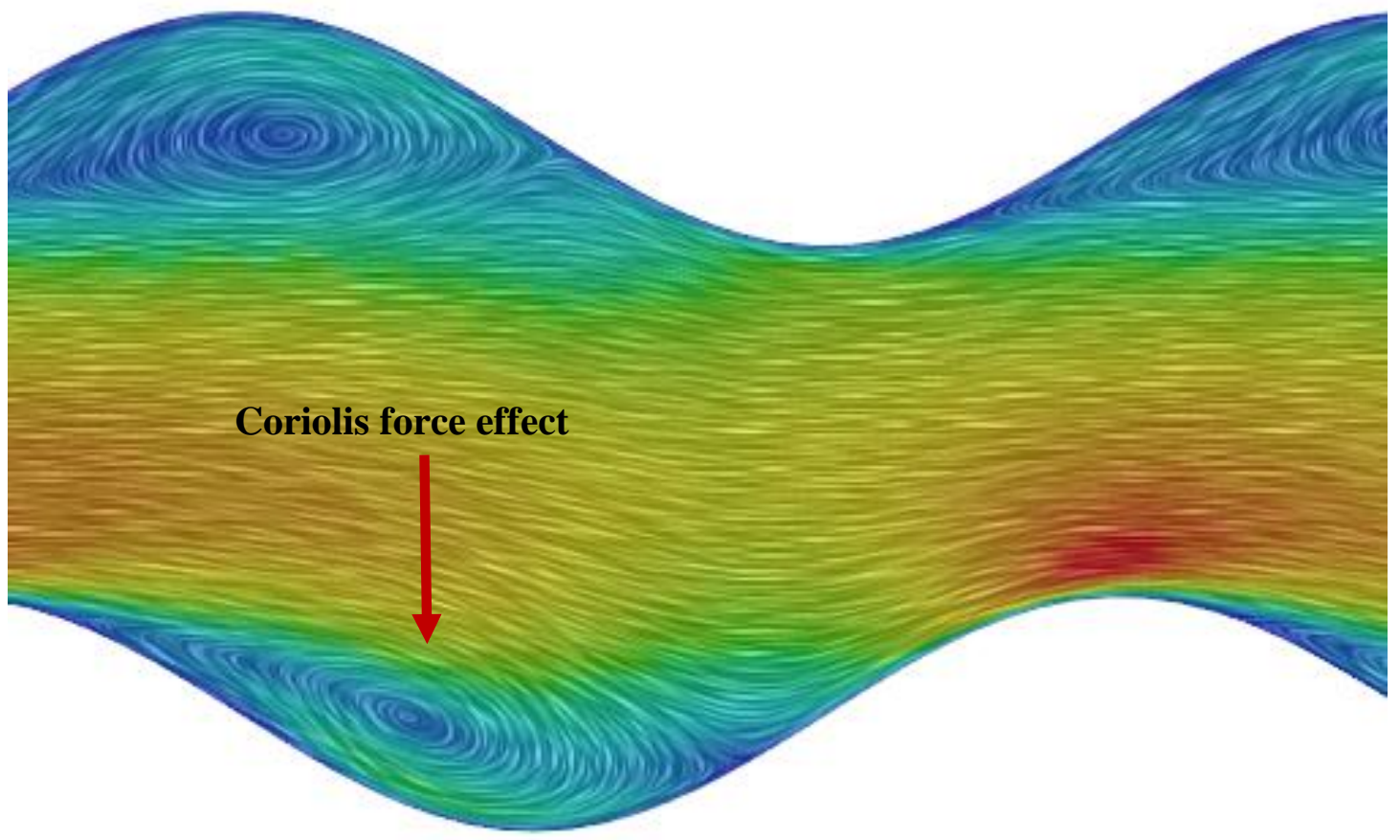

$\mathrm{Re}=4000$ and $1000 \mathrm{rpm}$

b) $\emptyset=90 \mathrm{deg}$ 

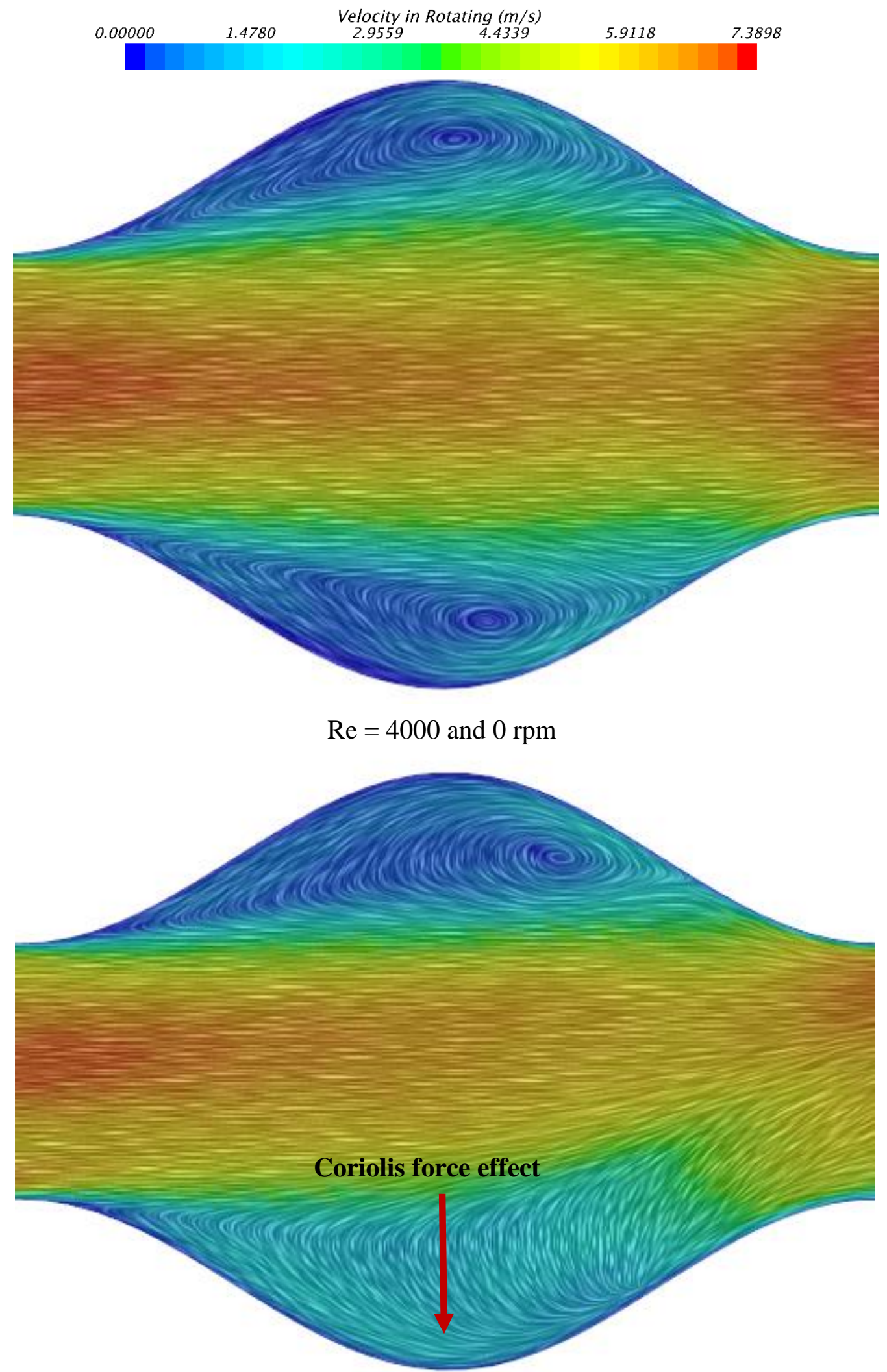

$$
\mathrm{Re}=4000 \text { and } 1000 \mathrm{rpm}
$$$$
\text { c) } \emptyset=180 \mathrm{deg}
$$

Figure 13: Velocity field at $Z=0$ for various shapes of the wavy channel at $R e=4000, \Omega=0$ and $1000 \mathrm{rpm}$. 
The isotherms distributions for the four different types of the channels at two different values of Reynolds number of $\operatorname{Re}=2000$ and 10000 and two different values of rotating speed of $\Omega=0$ and $1000 \mathrm{rpm}$ at $Z=0$ have also been presented in Figs. 14 and 15 . The figures reveal that the comparisons of the isotherms distributions clearly show that the wavy channel with $\phi=$ 0 generates greater temperature gradients in the regions along the upper and lower walls whereas the gradients are minimal for the channel with $\phi=180$. As a result, the heat transfer rate from the wavy channel with a phase-shift angle of $\phi=0$ is the highest compared to other geometries. A higher temperature gradient is clearly seen in the zones where greater shear wall stress exists or flow accelerates. The temperature field asymmetry is obvious from the distributions of the isotherms of the three different shapes of the wavy channel (Figs. 14 and 15a, c, e); the geometric asymmetry is the highest for the channel with a phase shift angle of $\phi=0$. The isotherms distribution for the channel with $\phi=0$ is found to be the most complex among the three channel configurations as shown in Figs. 14a and 15a.

When the channel is rotating, the flow accelerating downstream of the channel leads to increased streamwise velocity $(\mathrm{u})$ which in turn strengthens the magnitude of the negative $\mathrm{y}$ direction Coriolis force $(-2 \rho \Omega u)$. This causes the Coriolis force pointing to the lower wall and therefore the core of the mainstream fluid pushes toward the surface. As shown in Figs. 14 and $15 \mathrm{~b}, \mathrm{~d}, \mathrm{f}$, the effect of the Coriolis force $(-2 \rho \Omega u)$ is clearly visible (marked by red arrows) in the flow where the isotherms lines are pushed toward the lower wall. Consequently, more fluid from the core of the channel is forced to move against the wavy wall and it gets mixed with the hot flow close to the wall to enhance the heat transfer. Thus, the Nusselt number can be significantly enhanced on the lower wall as $\Omega$ increases. This is the reason why the lower wall Nusselt number raises rapidly as $\Omega$ increases. By comparing Fig. 14 with Fig. 15, the Coriolis force $(-2 \rho \Omega u)$ magnitude increases as Re raises due to an increase in the flow velocity component in the $\mathrm{x}$ direction. In this case, the Coriolis force $(-2 \rho \Omega u)$ may increase the flow patterns deflection towards the wall in the negative y-direction. 


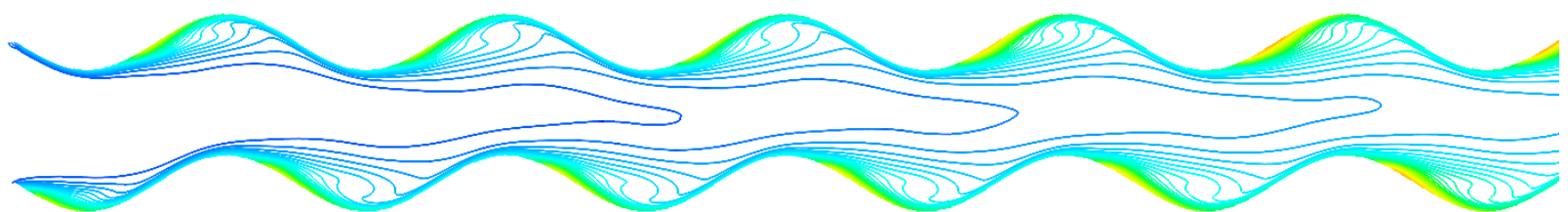

a) $\mathrm{Re}=2000,0 \mathrm{rpm}, \varnothing=0 \mathrm{deg}$

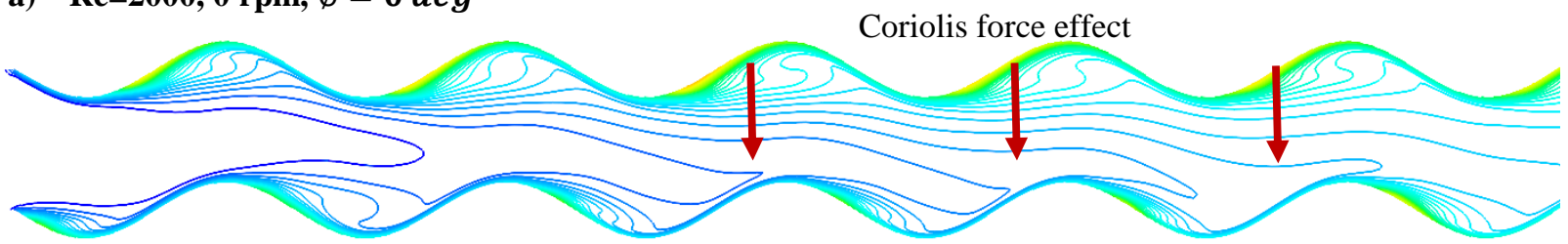

b) $\operatorname{Re}=2000,1000 \mathrm{rpm}, \varnothing=0 \mathrm{deg}$

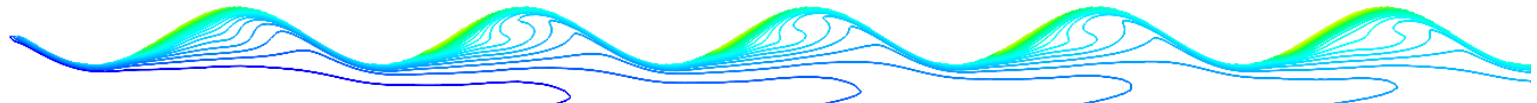

Temperature (K)

318
316
314
311
309
307
305
302
300
298

c) $\operatorname{Re}=2000,0 \mathrm{rpm}, \varnothing=90 \mathrm{deg}$

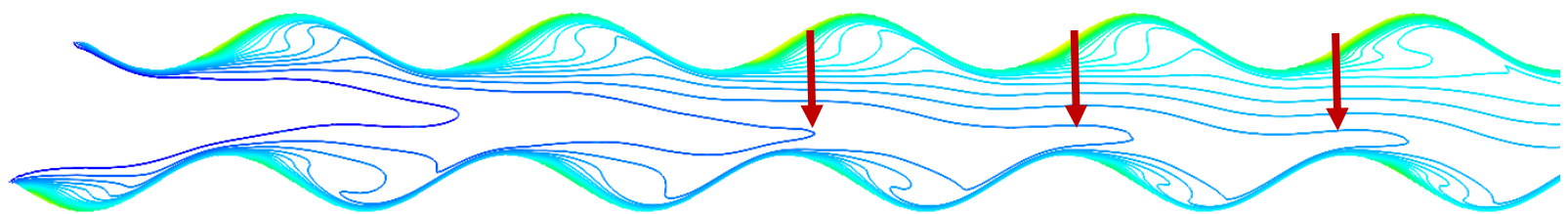

d) $\operatorname{Re}=2000,1000 \mathrm{rpm}, \varnothing=90 \mathrm{deg}$

e) $\operatorname{Re}=2000,0 \mathrm{rpm}, \emptyset=180 \mathrm{deg}$

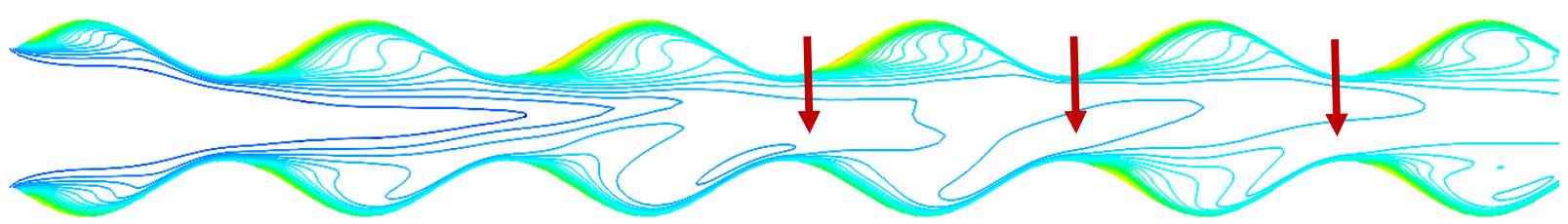

f) $\operatorname{Re}=2000,1000 \mathrm{rpm}, \varnothing=180 \mathrm{deg}$

g) $\operatorname{Re}=2000,0$ rpm, straight channel

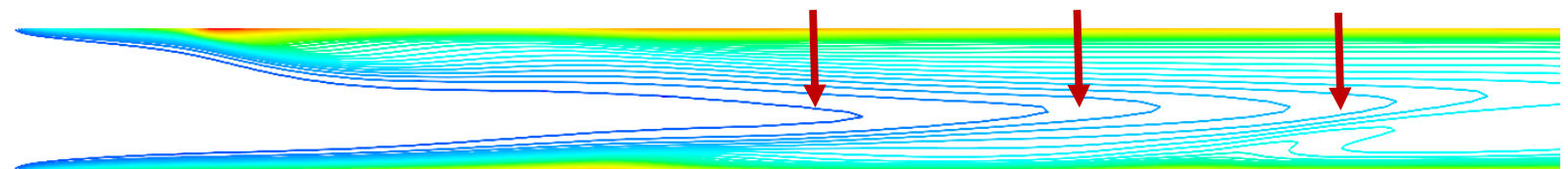

h) $\operatorname{Re}=2000,1000 \mathrm{rpm}$, straight channel

Figure 14: Temperature contours distributions at $Z=0$ for different channel geometries. 


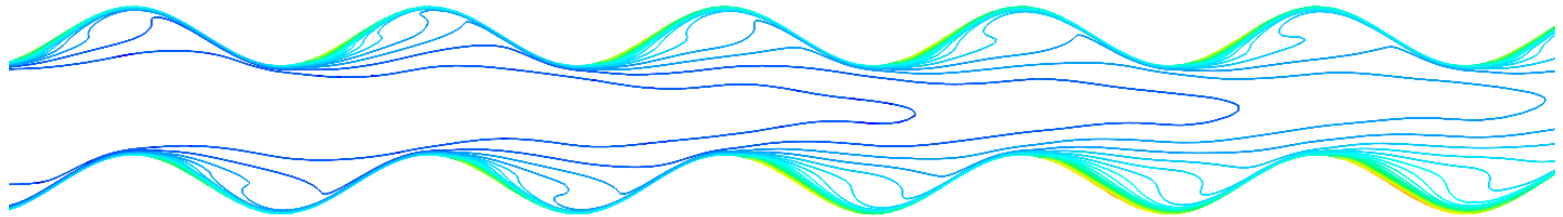

a) $\operatorname{Re}=10,000,0 \mathrm{rpm}, \emptyset=0 \mathrm{deg}$

Coriolis force effect

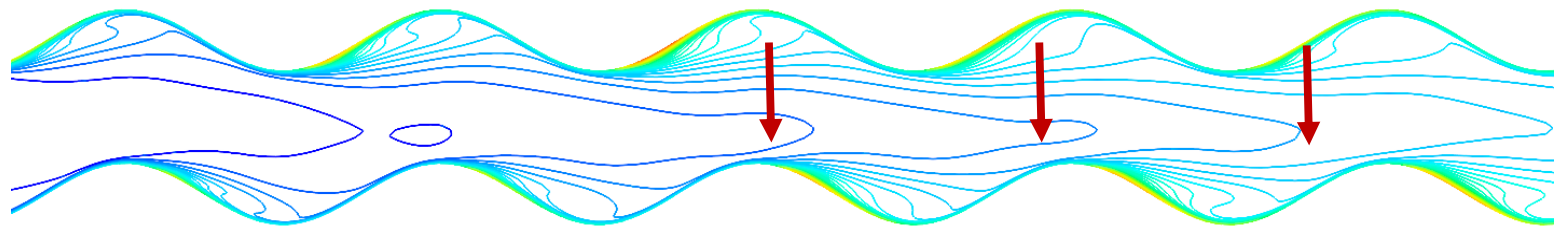

b) $R e=10,000,1000 \mathrm{rpm}, \emptyset=0 \mathrm{deg}$

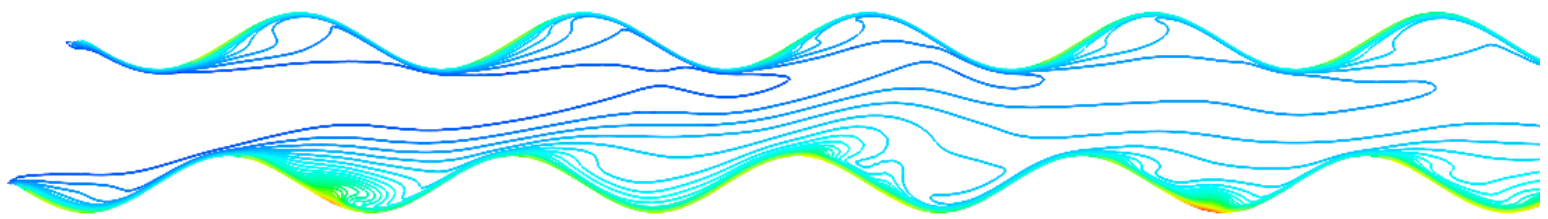

Temperature (K)

c) $\operatorname{Re}=10,000,0 \mathrm{rpm}, \emptyset=90 \mathrm{deg}$
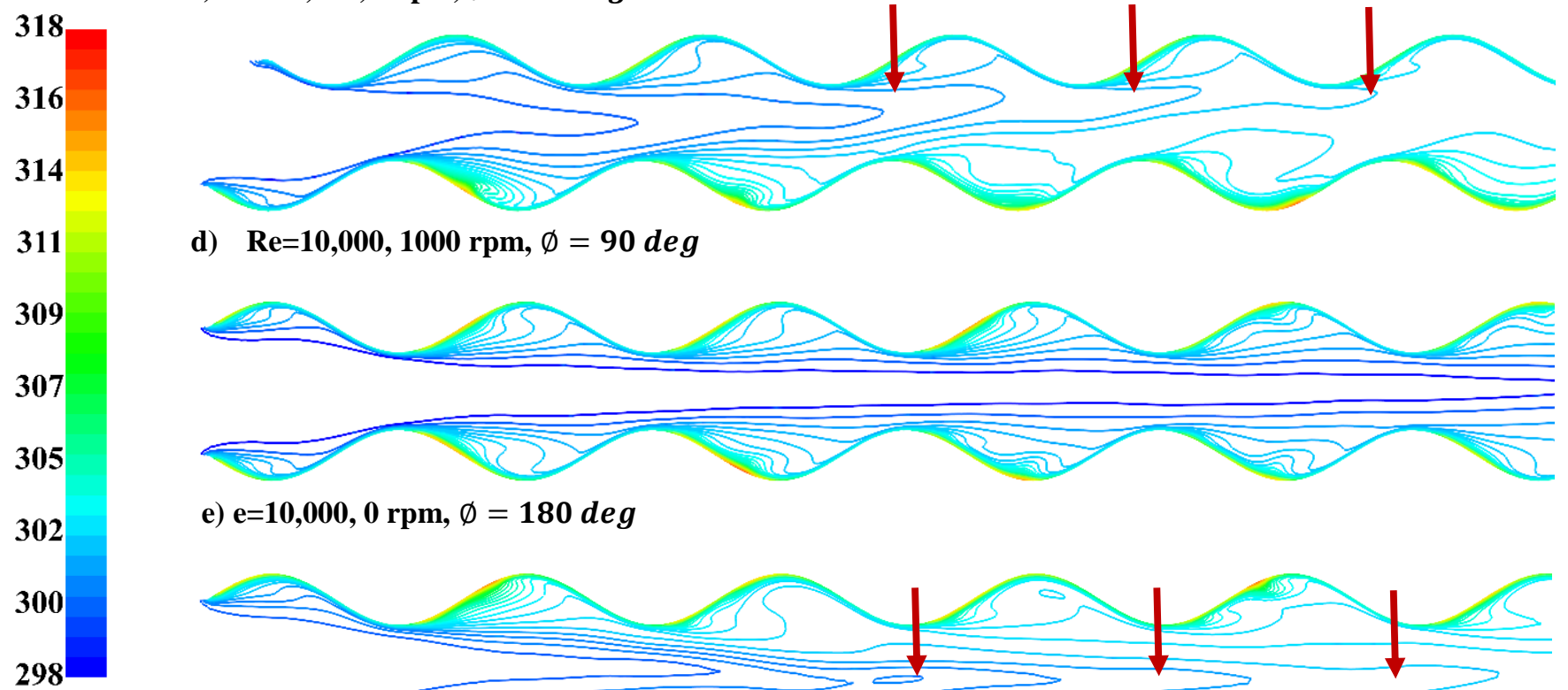

d) $\operatorname{Re}=10,000,1000 \mathrm{rpm}, \emptyset=90 \mathrm{deg}$

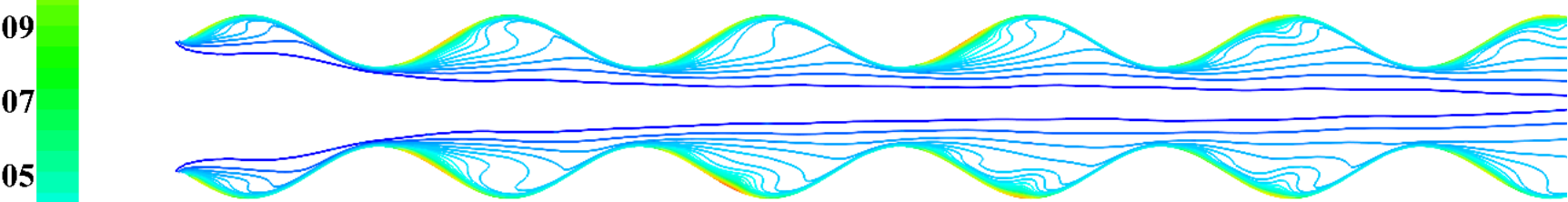

302 e) $\mathrm{e}=10,000,0 \mathrm{rpm}, \emptyset=180 \mathrm{deg}$

300

298

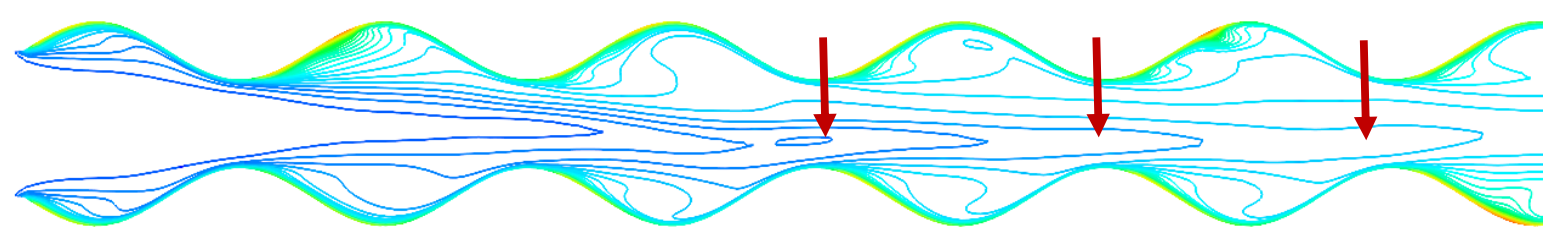

f) $\operatorname{Re}=10,000,1000 \mathrm{rpm}, \emptyset=180 \mathrm{deg}$

g) $\operatorname{Re}=10,000,0 \mathrm{rpm}$, straight channel
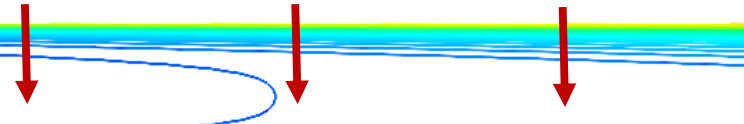

h) $R e=10,000,1000 \mathrm{rpm}$, straight channel

Figure 15: Temperature contours distributions at $\mathrm{Z}=0$ for different channel geometries. 
Fig. 16 illustrates the temperature distribution on the lower wall of the wavy channels with phase-shift angles of $\varnothing=0,90$, and 180 degrees at different values of Re and $\Omega$. The trace of the cooling fluid for the three cases of the stationary wavy channel is shorter and the temperature distribution on the lower wavy wall is nearly uniform because of the re-circulation zones which limiting the flow spreading in the spanwise direction. As Re increases, it is clearly seen that the cooling fluid shoots off into the core of the channel and therefore the spreading of the fluid takes place deep in the core instead of on the wavy walls. This, in turn, strengthens the cooling fluid attachment and the Nusselt number is enhanced accordingly.

The effect of rotation is also presented in Fig. 16. Under the rotational frame, the Coriolis force has a strong impact on the temperature distribution on the walls. As can be seen from this figure that increasing the rotating speed increases the Coriolis force $(-2 \rho \Omega u)$ effect which pushes the cooling fluid to the wall and thus it increases the flow spanwise spreading on the lower wall. On the other hand, increasing the rotating speed also increases the Coriolis force $(2 \rho \Omega v)$ effect which increases the flow streamwise spreading inside the channel. It is obvious that rotation has a substantial impact on the heat transfer enhancement because it can cause flow spreading in the spanwise direction and hence enhancing the spanwise heat transfer. 


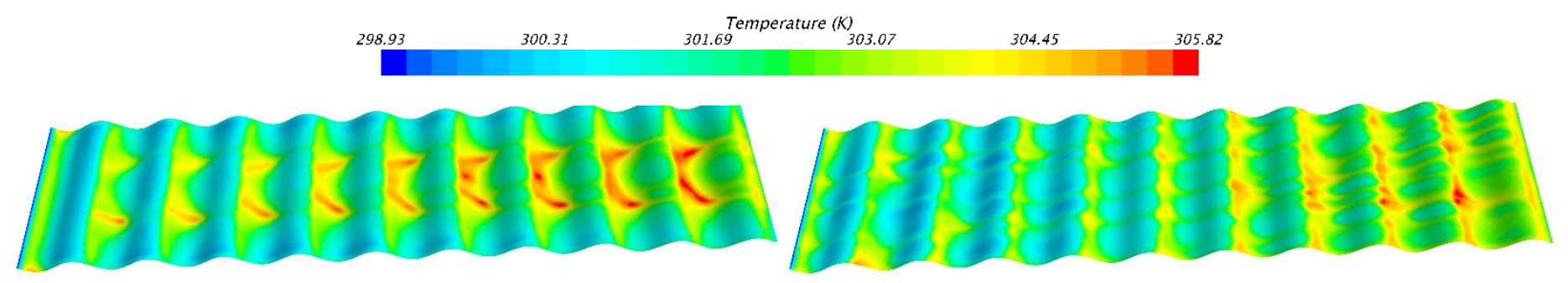

a) $\varnothing=0$ at $\mathrm{Re}=2000,0 \mathrm{rpm}$ and $1000 \mathrm{rpm}$

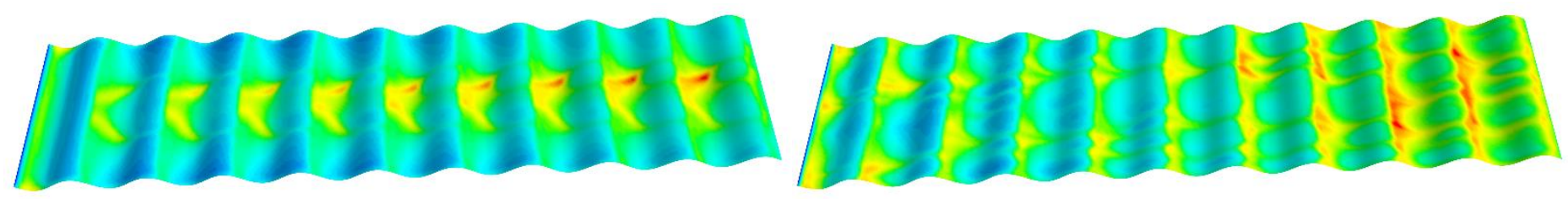

b) $\varnothing=90$ at $\mathrm{Re}=2000,0 \mathrm{rpm}$ and $1000 \mathrm{rpm}$

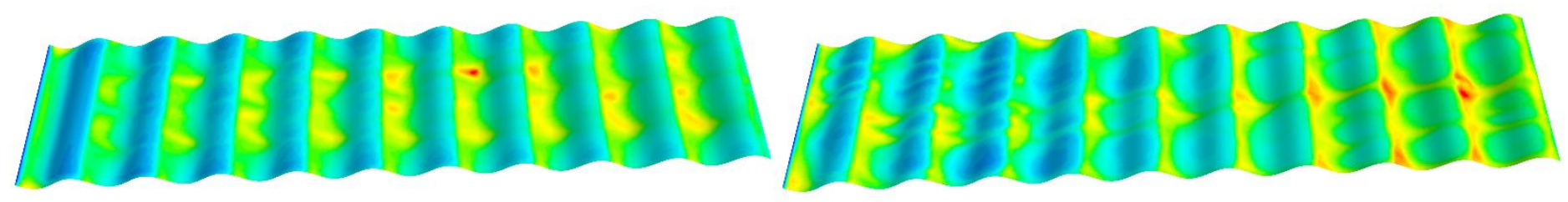

c) $\varnothing=180$ at $\mathrm{Re}=2000,0 \mathrm{rpm}$ and $1000 \mathrm{rpm}$

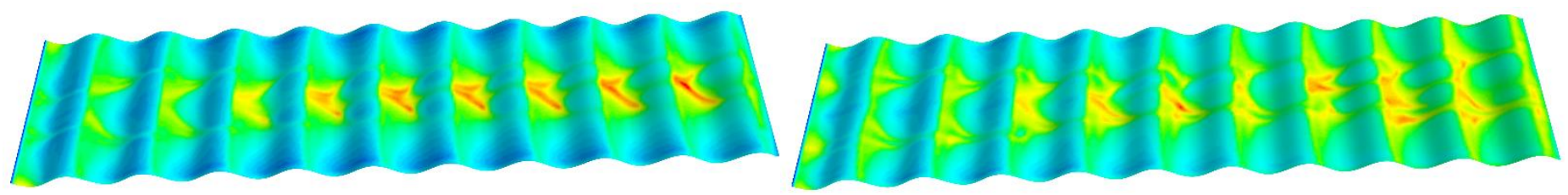

d) $\varnothing=0$ at $\mathrm{Re}=10000,0 \mathrm{rpm}$ and $1000 \mathrm{rpm}$

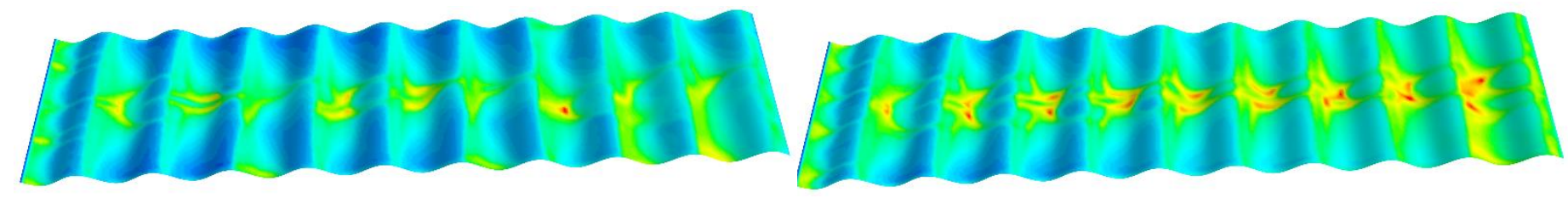

e) $\varnothing=90$ at $\mathrm{Re}=10000,0 \mathrm{rpm}$ and $1000 \mathrm{rpm}$

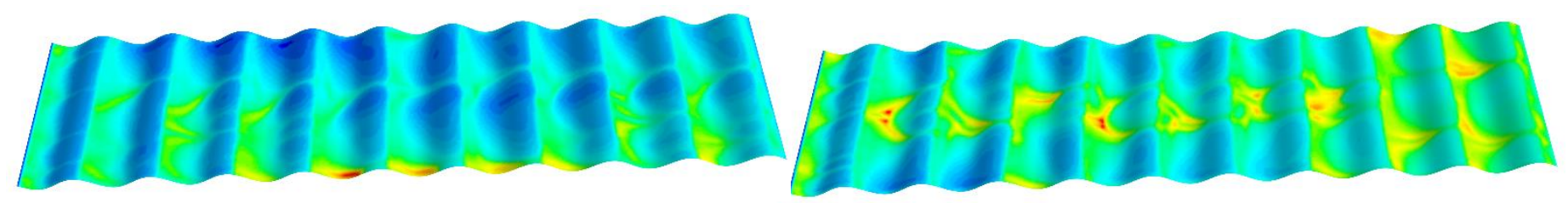

f) $\varnothing=180$ at $\mathrm{Re}=10000,0 \mathrm{rpm}$ and $1000 \mathrm{rpm}$

Figure 16: Temperature contours distributions on the lower wall for different shapes of channel geometries and different values of $\Omega$. 
The normalized Nusselt number $\left(N u_{a} / N u_{o}\right)$ is used to evaluate the performance of heat transfer from a surface [28], [29], [33].

Different correlations are employed to normalize the average Nusselt number in channel flows. In the current study, the correlation equation presented by Petukhov et al. [34] has been used to normalize the computed data and is defined as [28]:

$$
N u_{o}=\frac{\left(f_{o} / 8\right) \operatorname{RePr}}{1.07+12.7\left(f_{o} / 8\right)^{0.5}\left(\operatorname{Pr}^{2 / 3}-1\right)}
$$

Where:

- $\quad N u_{a}$ is the surface-averaged Nusselt number of the wavy wall calculated from equation 8.

- $N u_{o}$ is the Nusselt number computed from the correlation equation.

The computed friction factor $f_{o}$ is given by the Filonenko equation [28]:

$f_{o}=\left(1.82 \log _{10} R e-1.64\right)^{-2}$

It should be mentioned here that the above correlation equation (Eq.10) is applicable for $\operatorname{Re} \geq 10,000$. For values of Reynolds number in the range of $2.3 \times 10^{3} \leq R e \leq 10^{6}$ and Prandtl number in the range of $0.5 \leq \operatorname{Pr} \leq 200$ as in the present study, the modified form of Petukhov correlation (Eq. 10) has been used to normalize the average Nusselt number as follows [35] :

$$
N u_{o}=\frac{f_{o} / 8(R e-1000) \operatorname{Pr}}{1+12.7\left(f_{o} / 8\right)^{0.5}\left(\operatorname{Pr}^{2 / 3}-1\right)}
$$
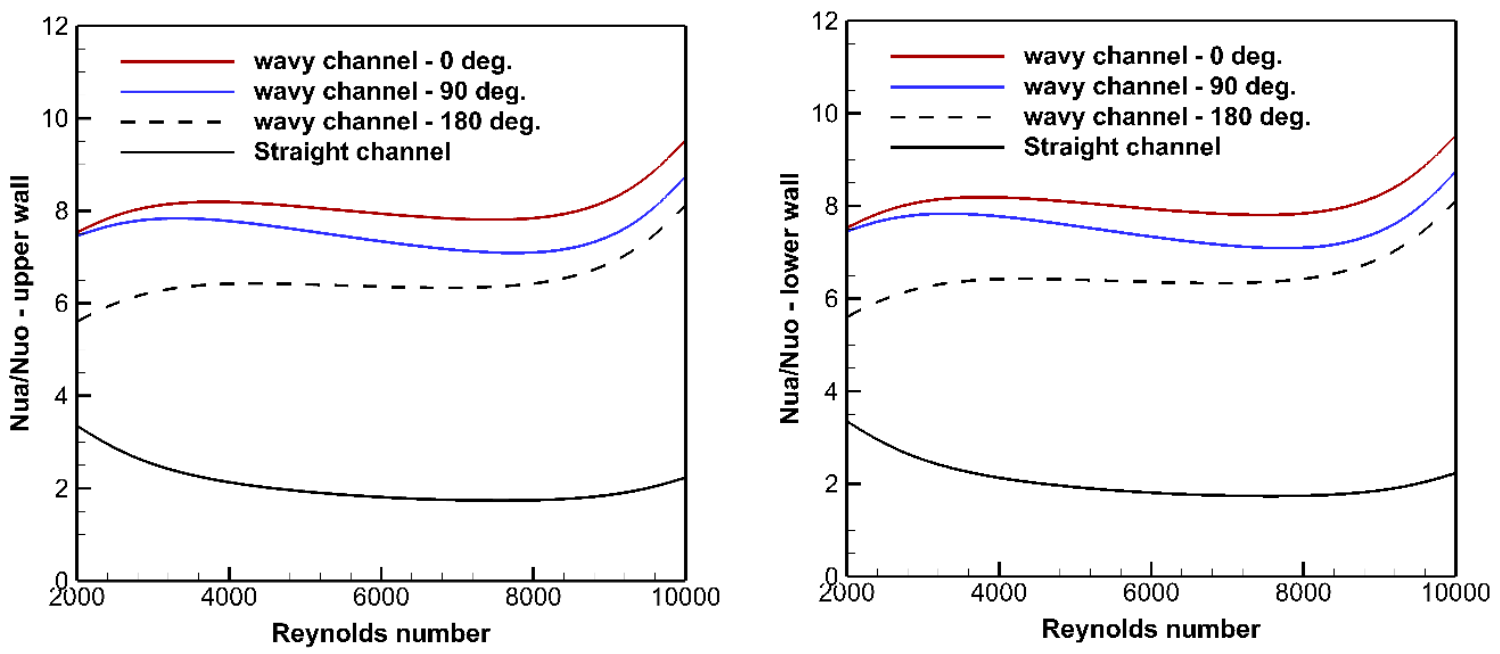

Figure 17: Normalized Nusselt number as a function of Reynolds number for various shapes of channels at $\Omega=0$ rpm. 

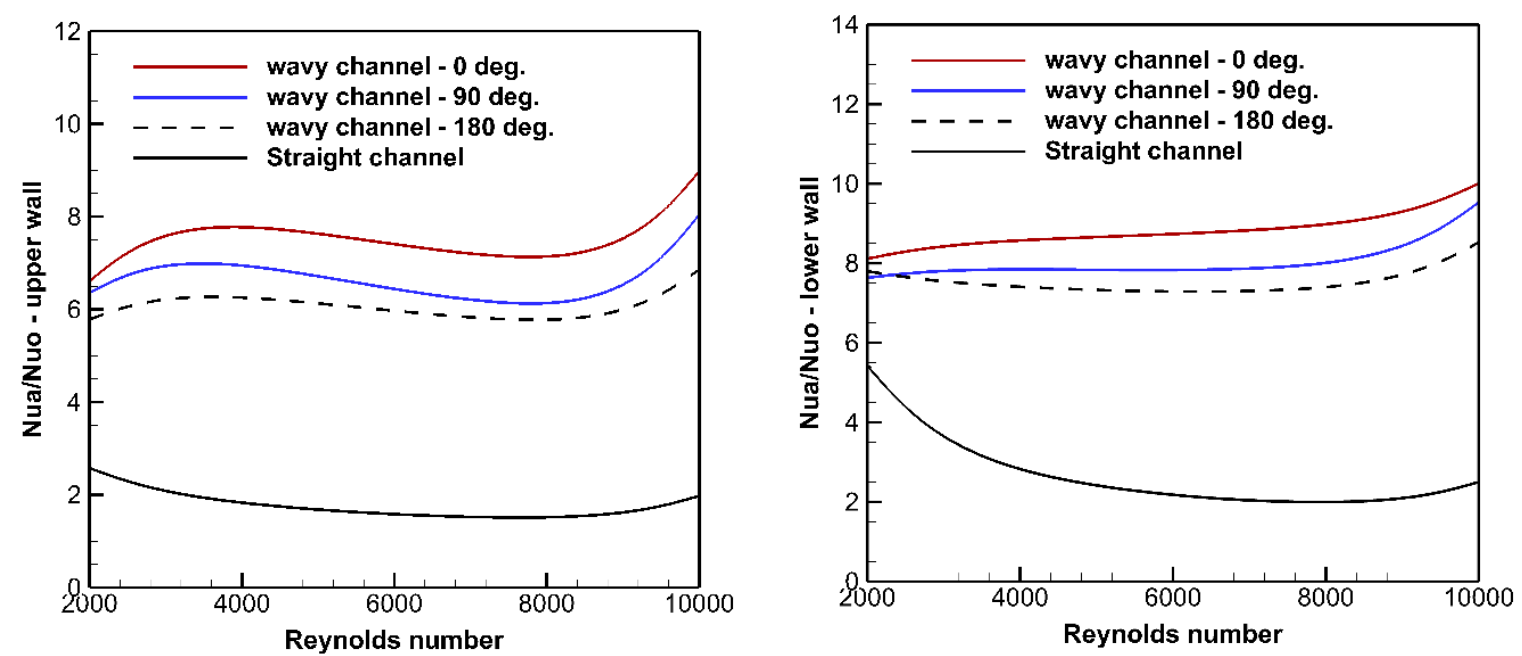

Figure 18: Normalized Nusselt number as a function of Reynolds number for various shapes of channels at $\Omega=500 \mathrm{rpm}$.
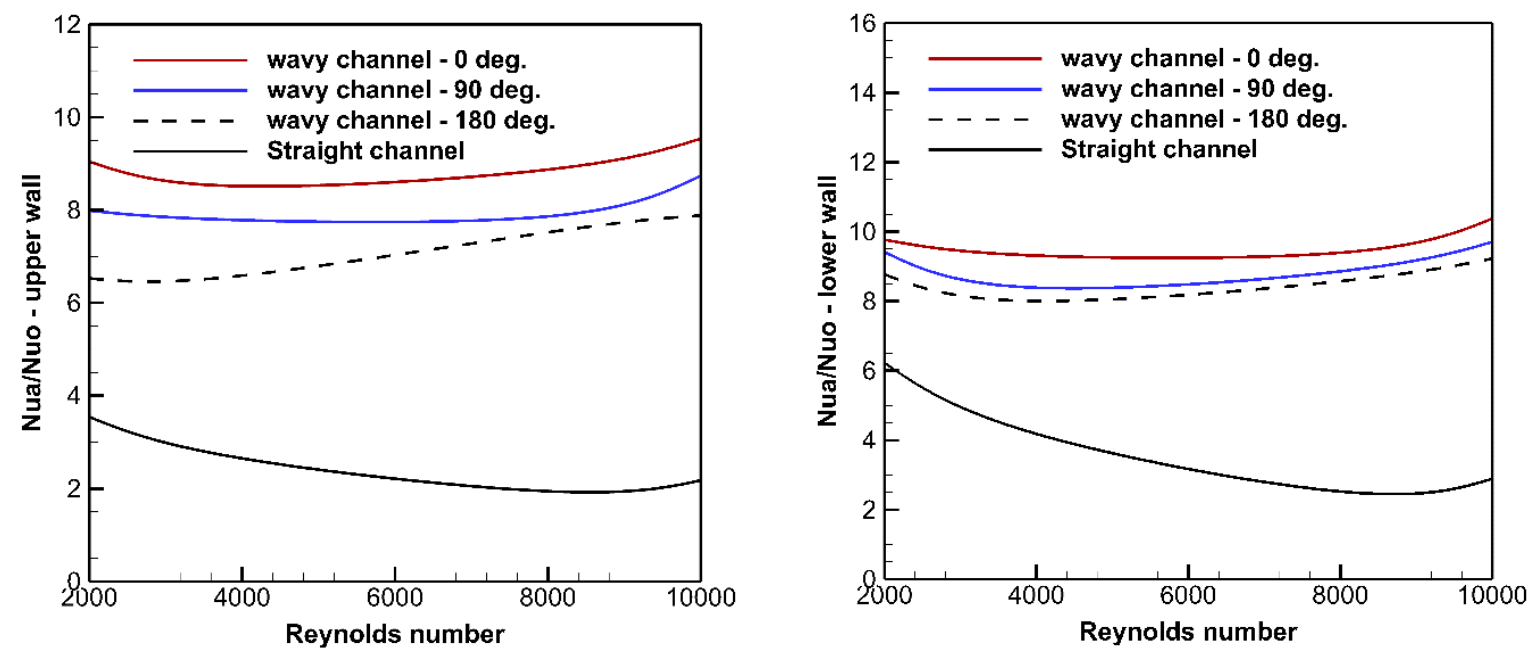

Figure 19: Normalized Nusselt number as a function of Reynolds number for various shapes of channels at $\Omega=1000$ rpm.

Figures 17-19 present the effect of Reynolds number on the normalized Nusselt number under different rotating speeds. Overall, the values of the $N u_{a} / N u_{o}$ ratio of the three geometries of the wavy channel increase with the increase of Reynolds number. Besides, they are higher than that in the case of a straight channel which has an inverse trend compared with that for the wavy channels. At $\Omega=0$ and $500 \mathrm{rpm}$, the ratios of $N u_{a} / N u_{o}$ are slightly affected by the change in Reynolds number. While they decrease with the increase in the value of Reynolds number for the case of a straight channel. It can be drawn from Figs. 17-19 that the phase-shift angle has a significant effect on the performance of the heat transfer enhancement of wavy surfaces.

In order to evaluate the performance of the heat transfer enhancement of the wavy surface, it is helpful to introduce the aerothermal efficiency $(\eta)$. It is a compromise between the heat transfer enhancement performance and friction loss when the heat transfer and associated pressure loss are both high [28], [29]. It can be defined as: 


$$
\eta=\frac{\frac{N u_{a}}{N u_{o}}}{\left(f / f_{o}\right)^{1 / 3}}
$$

It is obvious from figures 17-19 that the ratio of $N u_{a} / N u_{o}$ is proportional to Reynolds number while the friction factor is inversely proportional to Reynolds number. Thus, the aerothermal efficiency increases with the increase of Reynolds number, particularly when $\Omega>$ 0 as shown in Figs. 20-22. These figures show the aerothermal efficiency of the four geometries of the simulated channel at different Reynolds numbers and rotating speeds.

Furthermore, the results of the aerothermal efficiency with different rotating speeds at $\mathrm{Re}=4000$ and 10000 are presented in Fig. 23. The effects of the Coriolis force increase with the increase of rotating speed, influencing the performance of heat transfer enhancement by affecting the air flow characteristics inside the channel. It was observed from Fig. 23 that at $\mathrm{Re}=4000$, the aerothermal efficiency decreases with the increase of rotating speed, while it is parabolic with the increase of rotating speed at $\operatorname{Re}=10000$. It was also shown that the aerothermal efficiency of the straight channel rapidly drops as the rotating speed increases compared with that of the wavy channel. This indicates that the wavy channels provide a higher heat transfer performance for different values of Reynolds number and rotating speed than that of a straight channel.
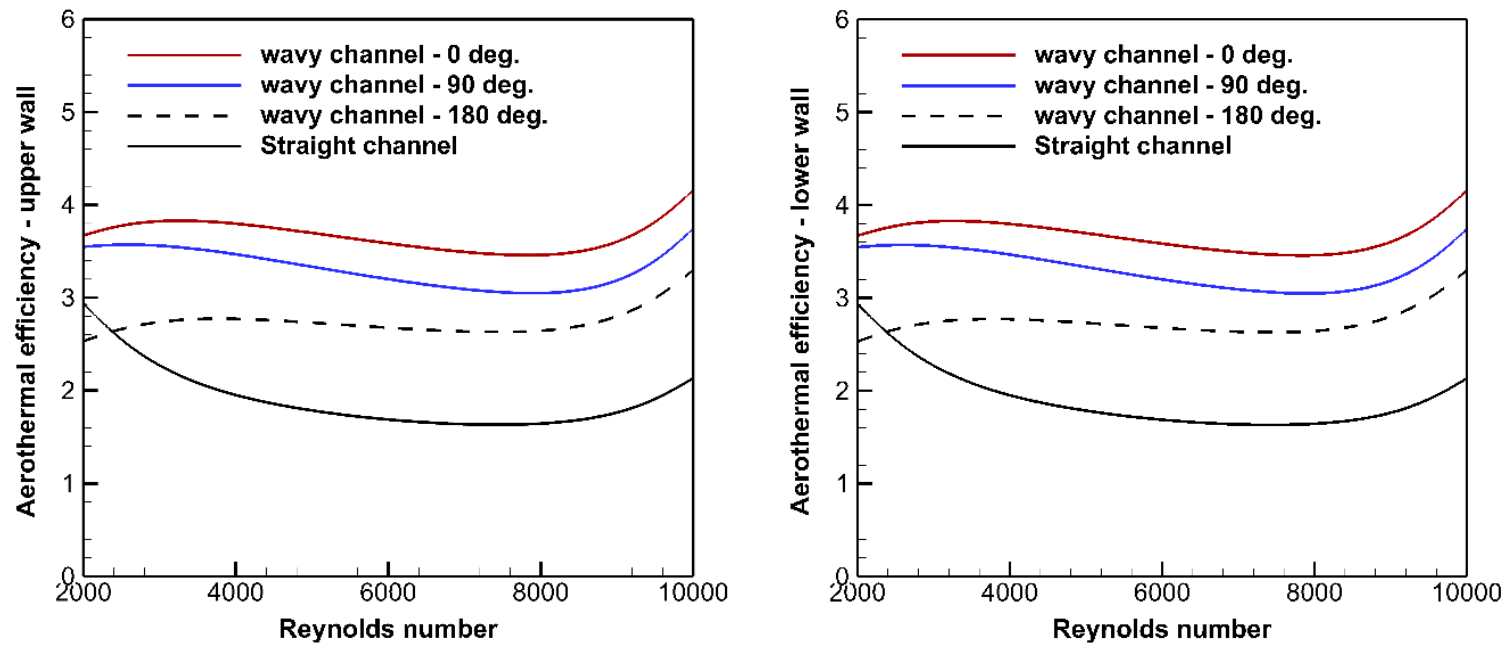

Figure 20: Aerothermal efficiency as a function of Reynolds number for various shapes of channels at $\Omega=$ 0 rpm. 

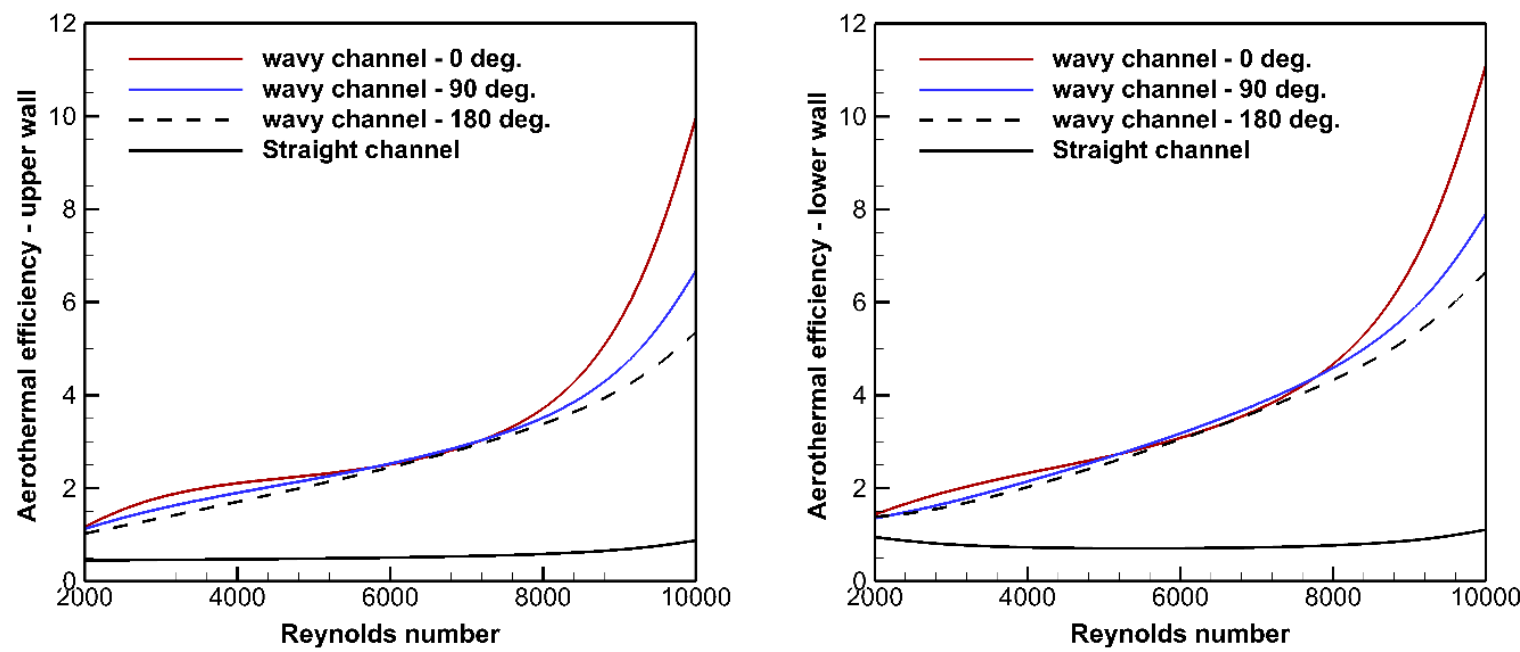

Figure 21: Aerothermal efficiency as a function of Reynolds number for various shapes of channels at $\Omega=$ 500 rpm.
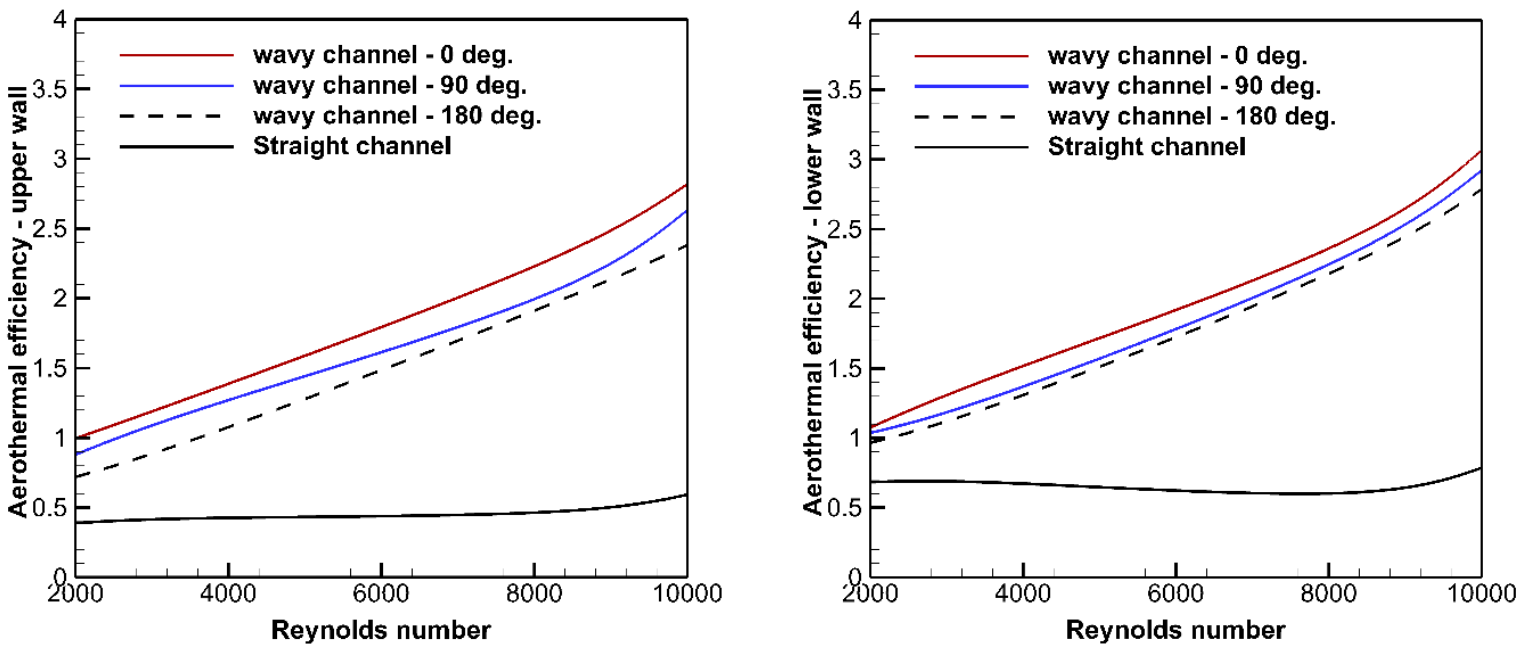

Figure 22: Aerothermal efficiency as a function of Reynolds number for various shapes of channels at $\Omega=$ $1000 \mathrm{rpm}$. 


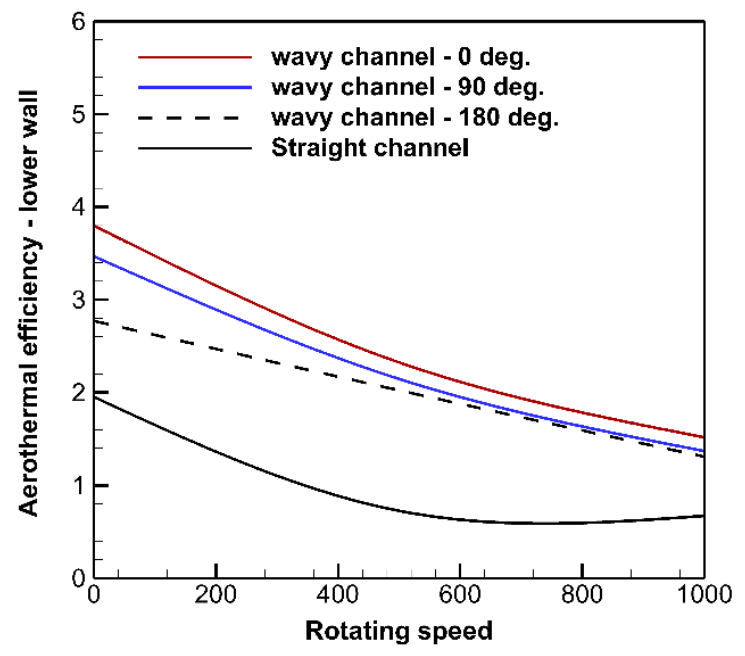

a) $\mathrm{Re}=4000$

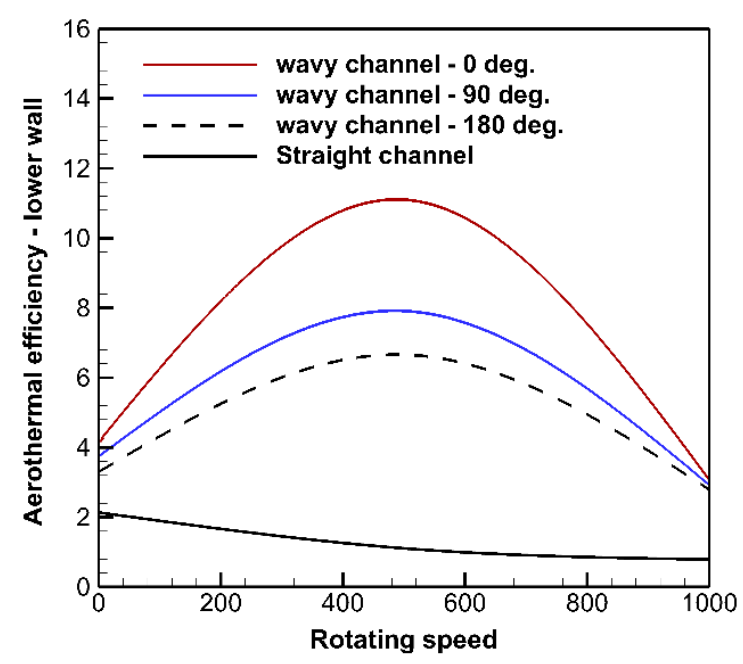

b) $\mathrm{Re}=10000$

Figure 23: The effect of rotating speed on the aerothermal efficiency at different values of Reynolds number for the lower wall.

\section{9-Conclusions:}

In this paper, flow and heat transfer performance in different types of channels geometries at stationary and rotating conditions were investigated numerically for a wide range of Reynolds number of 1000-10000 and a rotating speed varying from 0 to $1000 \mathrm{rpm}$. The wavy surface profile was created using a phase difference between the upper and lower walls. The significant impact of the rotation on the performance of wavy channels, including the flow improvement and heat transfer enhancement was analyzed and discussed. The highlights of the present study are given below:

- For a given value of Re, the Nusselt number increases as the rotating speed increases for all shapes of the channel. For the same value of rotating speed, it was found that the enhancement in heat transfer depends on Re value.

- The fluctuations in the values of the local Nusselt number along the streamwise direction is due to the curvature effect. The maximum value of Nu occurs right at the minimal crosssection. On the other hand, the minimum value of $\mathrm{Nu}$ occurs nearly upstream of the maximized cross-section region.

- Rotation has a strong impact on the flow field and heat transfer performance. As the rotating speed increases, the Coriolis force further promotes the heat transfer on the lower wall. The heat transfer rate from the upper wall is directly proportional to the rotating speed owing to the mainstream fluid impingement. Furthermore, as Re increases, the isotherm lines move toward the lower wavy wall and $\mathrm{Nu}$ and heat exchange increase.

- Flow structures were observed to be complicated for the three different channel geometries along with a greater wall temperature gradient was clearly seen for the channel with $\emptyset=$ $0 \mathrm{deg}$. 
- In addition, it was observed that the wavy channel with a phase shift angle of $\varnothing=0$ deg produces the highest surface-averaged Nusselt number and heat transfer enhancement in comparison to other geometries but it is accompanied by a relatively high pressure drop penalty.

\section{Acknowledgements}

The assistance given by IT Services and the use of the Computational Shared Facility at the University of Manchester-UK, are greatly appreciated. Also, the support presented by the University of Kufa - Faculty of Engineering - the Kufa Centre for Advanced Simulation in Engineering "KCASE" is greatly acknowledged.

\section{References}

[1] D. Junqi, C. Jiangping, C. Zhijiu, Z. Yimin, and Z. Wenfeng, "Heat transfer and pressure drop correlations for the wavy fin and flat tube heat exchangers," Appl. Therm. Eng., vol. 27, no. 11-12, pp. 2066-2073, Aug. 2007.

[2] L. Goldstein and E. M. Sparrow, "Heat/Mass Transfer Characteristics for Flow in a Corrugated Wall Channel," J. Heat Transfer, vol. 99, no. 2, p. 187, 1977.

[3] C.-C. Wang and C.-K. Chen, "Forced convection in a wavy-wall channel," Int. J. Heat Mass Transf., vol. 45, no. 12, pp. 2587-2595, Jun. 2002.

[4] N. Tatsuo, M. Shinichiro, A. Shingho, and K. Yuji, "Flow observations and mass transfer characteristics in symmetrical wavy-walled channels at moderate Reynolds numbers for steady flow," Int. J. Heat Mass Transf., vol. 33, no. 5, pp. 835-845, May 1990.

[5] D. R. Sawyers, M. Sen, and H.-C. Chang, "Heat transfer enhancement in three-dimensional corrugated channel flow," Int. J. Heat Mass Transf., vol. 41, no. 22, pp. 3559-3573, Nov. 1998.

[6] T. a. Rush, T. a. Newell, and A. m. Jacobi, "An experimental study of flow and heat transfer in sinusoidal wavy passages,” Int. J. Heat Mass Transf., vol. 42, no. 9, pp. 1541-1553, May 1999.

[7] G. Fabbri, "Heat transfer optimization in corrugated wall channels," Int. J. Heat Mass Transf., vol. 43, no. 23, pp. 4299-4310, Dec. 2000.

[8] G. Fabbri and R. Rossi, "Analysis of the heat transfer in the entrance region of optimised corrugated wall channel,” Int. Commun. Heat Mass Transf., vol. 32, no. 7, pp. 902-912, Jul. 2005.

[9] P. Naphon, "Laminar convective heat transfer and pressure drop in the corrugated channels," Int. Commun. Heat Mass Transf., vol. 34, no. 1, pp. 62-71, Jan. 2007.

[10] P. Naphon, "Effect of corrugated plates in an in-phase arrangement on the heat transfer and flow developments," Int. J. Heat Mass Transf., vol. 51, no. 15-16, pp. 3963-3971, Jul. 2008.

[11] P. Naphon, "Effect of wavy plate geometry configurations on the temperature and flow distributions," Int. Commun. Heat Mass Transf., vol. 36, no. 9, pp. 942-946, Nov. 2009.

[12] H. M. Metwally and R. M. Manglik, "Enhanced heat transfer due to curvature-induced lateral vortices in laminar flows in sinusoidal corrugated-plate channels," Int. J. Heat Mass Transf., vol. 47, no. 10-11, pp. 2283-2292, May 2004.

[13] G.-N. Xie, Q.-W. Wang, M. Zeng, and L.-Q. Luo, "Numerical investigation of heat transfer and fluid flow characteristics inside a wavy channel," Heat Mass Transf., vol. 43, no. 7, pp. 603-611, Mar. 2007. 
[14] M. Z. Hossain and A. K. M. S. Islam, "FULLY DEVELOPED FLOW STRUCTURES AND HEAT TRANSFER IN SINE-SHAPED WAVY CHANNELS," Int. Commun. Heat Mass Transf., vol. 31, no. 6, pp. 887-896, Aug. 2004.

[15] B. Ničeno and E. Nobile, "Numerical analysis of fluid flow and heat transfer in periodic wavy channels," Int. J. Heat Fluid Flow, vol. 22, no. 2, pp. 156-167, Apr. 2001.

[16] H. M. S. Bahaidarah, N. K. Anand, and H. C. Chen, "NUMERICAL STUDY OF HEAT AND MOMENTUM TRANSFER IN CHANNELS WITH WAVY WALLS," Numer. Heat Transf. Part A Appl., vol. 47, no. 5, pp. 417-439, Mar. 2005.

[17] A. G. Ramgadia and A. K. Saha, "Characteristics of Fully Developed Flow and Heat Transfer in Channels With Varying Wall Geometry,” J. Heat Transfer, vol. 136, no. 2, p. 021703, Nov. 2013.

[18] A. G. Ramgadia and A. K. Saha, "Numerical study of fully developed unsteady flow and heat transfer in asymmetric wavy channels," Int. J. Heat Mass Transf., vol. 102, pp. 98-112, Nov. 2016.

[19] F. Oviedo-Tolentino, R. Romero-Méndez, A. Hernández-Guerrero, and B. Girón-Palomares, "Experimental study of fluid flow in the entrance of a sinusoidal channel," Int. J. Heat Fluid Flow, vol. 29, no. 5, pp. 12331239, Oct. 2008.

[20] H. M. S. Bahaidarah, "A Numerical Study of Fluid Flow and Heat Transfer Characteristics in Channels with Staggered Wavy Walls," Numer. Heat Transf. Part A Appl., vol. 51, no. 9, pp. 877-898, Apr. 2007.

[21] T. Nishimura and S. Matsune, "Mass transfer enhancement in a sinusoidal wavy channel for pulsatile flow," Heat Mass Transf., vol. 32, no. 1-2, pp. 65-72, Nov. 1996.

[22] S. Mahmud, A. Sadrul Islam, and C. Feroz, "Flow and heat transfer characteristics inside a wavy tube," Heat Mass Transf., vol. 39, no. 5, pp. 387-393, Jun. 2003.

[23] L. Zhang and D. Che, "Influence of Corrugation Profile on the Thermalhydraulic Performance of CrossCorrugated Plates," Numer. Heat Transf. Part A Appl., vol. 59, no. 4, pp. 267-296, Feb. 2011.

[24] H. A. Mohammed, P. Gunnasegaran, and N. H. Shuaib, "Numerical simulation of heat transfer enhancement in wavy microchannel heat sink," Int. Commun. Heat Mass Transf., vol. 38, no. 1, pp. 63-68, Jan. 2011.

[25] M. A. Ahmed, M. Z. Yusoff, K. C. Ng, and N. H. Shuaib, "Numerical and experimental investigations on the heat transfer enhancement in corrugated channels using SiO2-water nanofluid," Case Stud. Therm. Eng., vol. 6, pp. 77-92, Sep. 2015.

[26] H K Versteeg and W Malalasekera, An Introduction to Computational Fluid Dynamics, Second Edi. England: Pearson education, 2007.

[27] J. Y. Luo, R. I. Issa, and A. D. Gosman, "Prediction of Impeller- Induced Flows in Mixing Vessels Using Multiple Frames of Reference," Inst. Chem. Eng. Symp. Ser., vol. 136, pp. 549-556, 1994.

[28] W. Siddique, L. El-Gabry, I. V. Shevchuk, N. B. Hushmandi, and T. H. Fransson, "Flow structure, heat transfer and pressure drop in varying aspect ratio two-pass rectangular smooth channels," Heat Mass Transf., vol. 48, no. 5, pp. 735-748, May 2012.

[29] I. V. Shevchuk, S. C. Jenkins, B. Weigand, J. von Wolfersdorf, S. O. Neumann, and M. Schnieder, "Validation and Analysis of Numerical Results for a Varying Aspect Ratio Two-Pass Internal Cooling Channel," J. Heat Transfer, vol. 133, no. 5, May 2011.

[30] S. . Patankar and D. . Spalding, "A calculation procedure for heat, mass and momentum transfer in threedimensional parabolic flows," Int. J. Heat Mass Transf., vol. 15, no. 10, pp. 1787-1806, Oct. 1972.

[31] A. Fasquelle, J. Pellé, S. Harmand, and I. V. Shevchuk, "Numerical Study of Convective Heat Transfer Enhancement in a Pipe Rotating Around a Parallel Axis," J. Heat Transfer, vol. 136, no. 5, May 2014.

[32] C. Zhang, Y. Niu, and J. Xu, "An anisotropic turbulence model for predicting heat transfer in a rotating 
channel,” Int. J. Therm. Sci., vol. 148, p. 106119, Feb. 2020.

[33] W. Siddique, I. V. Shevchuk, L. El-Gabry, N. B. Hushmandi, and T. H. Fransson, "On flow structure, heat transfer and pressure drop in varying aspect ratio two-pass rectangular channel with ribs at $45^{\circ}$," Heat Mass Transf., vol. 49, no. 5, pp. 679-694, May 2013.

[34] Petukhov BS, Irvine TF, and Hartnett JP, Advances in heat transfer, Vol 6. New York: Academic Press, 1970.

[35] D. Taler, "Experimental determination of correlations for average heat transfer coefficients in heat exchangers on both fluid sides," Heat Mass Transf., vol. 49, no. 8, pp. 1125-1139, Aug. 2013. 
We certify that the present manuscript has not been published in an archival journal. Also, we certify that the present manuscript is not currently submitted for publication in another journal.

Authors 


\section{AUTHORSHIP STATEMENT}

Manuscript title: Effect of rotation on forced convection in wavy wall channels

All persons who meet authorship criteria are listed as authors, and all authors certify that they have participated sufficiently in the work to take public responsibility for the content, including participation in the concept, design, analysis, writing, or revision of the manuscript. Furthermore, each author certifies that this material or similar material has not been and will not be submitted to or published in any other publication before its appearance in the International Journal of Heat and Mass Transfer.

\section{$\underline{\text { Authorship contributions }}$}

Please indicate the specific contributions made by each author (list the authors' initials followed by their surnames, e.g., Y.L. Cheung). The name of each author must appear at least once in each of the three categories below.

\section{- Category 1}

Conceptualization: N. M. Al-Zurfi, A. N. Alhusseny, and A. G. Nasser

Acquisition of data: N. M. Al-Zurfi, A. N. Alhusseny, and A. G. Nasser

Methodology: N. M. Al-Zurfi, A. N. Alhusseny, and A. G. Nasser

Data curation: N. M. Al-Zurfi, and A. N. Alhusseny

Software: N. M. Al-Zurfi, and A. N. Alhusseny

Validation: N. M. Al-Zurfi

Resources: A. G. Nasser

Supervision: N. M. Al-Zurfi, and A. G. Nasser

Project administration: N. M. Al-Zurfi

\section{- Category 2}

Writing- Original draft preparation: N. M. Al-Zurfi, A. N. Alhusseny, and A. G. Nasser Revising the manuscript critically for important intellectual content: N. M. Al-Zurfi 


\section{- Category 3}

Approval of the version of the manuscript to be published (the names of all authors must be listed):

N. M. Al-Zurfi, A. N. Alhusseny, and A. G. Nasser

This statement is signed by all the authors:

Author's name (typed)

Nabeel Al-Zurfi

Ahmed Alhusseny

Adel Nasser
Author's signature
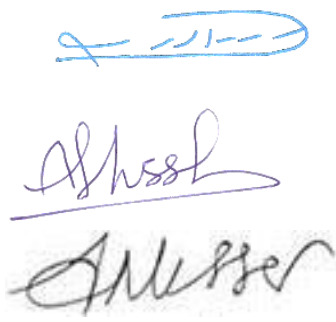

Date

$30 / 11 / 2019$

$30 / 11 / 2019$

$30 / 11 / 2019$ 